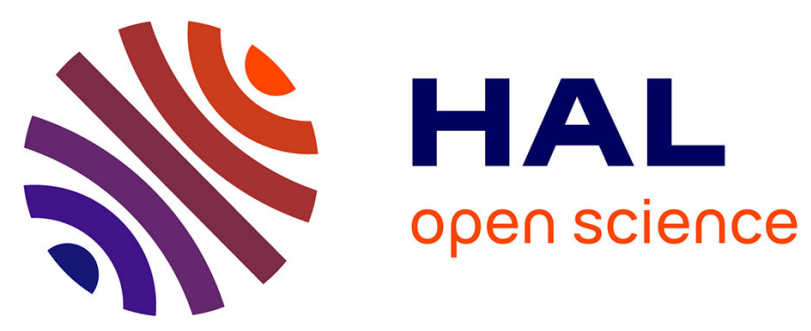

\title{
Stable Impulsive Zone MPC for Type 1 Diabetic Patients based on a long-term model
}

Alejandro Hernan Gonzalez, Pablo S. Rivadeneira, Antonio Ferramosca, Nicolas Magdelaine, Claude H. Moog

\section{To cite this version:}

Alejandro Hernan Gonzalez, Pablo S. Rivadeneira, Antonio Ferramosca, Nicolas Magdelaine, Claude H. Moog. Stable Impulsive Zone MPC for Type 1 Diabetic Patients based on a long-term model. Optimal Control Applications and Methods, 2020, Control for Hybrid Systems: Applications and Methods for Adaptation and Optimality, 41 (6), pp.2115-2136. 10.1002/oca.2647 . hal-02660136

\section{HAL Id: hal-02660136 https://hal.science/hal-02660136}

Submitted on 2 Dec 2020

HAL is a multi-disciplinary open access archive for the deposit and dissemination of scientific research documents, whether they are published or not. The documents may come from teaching and research institutions in France or abroad, or from public or private research centers.
L'archive ouverte pluridisciplinaire HAL, est destinée au dépôt et à la diffusion de documents scientifiques de niveau recherche, publiés ou non, émanant des établissements d'enseignement et de recherche français ou étrangers, des laboratoires publics ou privés. 
DOI: $\mathrm{xxx} / \mathrm{xxxx}$

\title{
RESEARCH ARTICLE
}

\section{Stable Impulsive Zone MPC for Type 1 Diabetic Patients based on a long-term model}

\author{
Alejandro H. González*1 | Pablo S. Rivadeneira ${ }^{2}$ | Antonio Ferramosca ${ }^{3}$ | Nicolas \\ Magdelaine $^{4}$ | Claude H. Moog ${ }^{4}$
}

\author{
${ }^{1}$ Institute of Technological Development for \\ the Chemical Industry (INTEC),CONICET- \\ Universidad Nacional del Litoral (UNL). \\ Güemes 3450, (3000) Santa Fe, Argentina. \\ ${ }^{2}$ Universidad Nacional de Colombia, \\ Facultad de Minas, Grupo GITA, Cra. 80 \\ $\mathrm{N}^{\circ}$ 65-223, Medellín, Colombia. \\ ${ }^{3}$ CONICET - Universidad Tecnológica \\ Nacional (UTN), Facultad Regional de \\ Reconquista. 27 de Abril 1000 (3560), \\ Reconquista, Santa Fe, Argentina. \\ ${ }^{4}$ LS2N, UMR CNRS 6004, BP 92101, \\ 44321 Nantes Cedex 3, France. \\ Correspondence \\ *Alejandro H. González. Email: \\ alejgon@santafe-conicet.gov.ar
}

\begin{abstract}
In this work, the problem of regulating blood glucose (glycemia) in type I diabetic patients is studied by means of an impulsive zone model predictive control (iZMPC), which bases its predictions on a novel long-term glucose-insulin model. Taking advantage of the impulsive version of the model - which features real life properties of diabetes patients that some other popular models do not - the given control guarantees the stability under moderate-to-severe plant-model mismatch and disturbances. Long-term scenarios - including meals and physiological parameter variations - are simulated and the results are satisfactory as every hyperglycemic and hypoglycemic episodes are suitably controlled.
\end{abstract}

\section{KEYWORDS:}

Impulsive control systems; Type I diabetes model; Zone model predictive control.

\section{1 | INTRODUCTION}

Type 1 diabetes mellitus (T1DM) is an autoimmune disease, affecting approximately 18 million people in the world. It is characterized by the destruction of the pancreatic $\beta$-cells, which avoids the natural endogenous secretion of insulin, thus resulting in a dysfunctional glycemic regulation. T1DM was a fatal disease until the discovery of insulin in 1921. Nowadays, the current treatment consists of a number of daily insulin injections, depending on the measurements of glycemia and carbohydrate intake. The exogenous insulin injections may be provided by a pump that uses both, a permanent basal subcutaneous infusion and preprandial boluses (CSII). In any case, the objective is to maintain glycemia in a safe zone, between $80 \mathrm{mg} / \mathrm{dl}$ and $140 \mathrm{mg} / \mathrm{dl}^{1}$, the so-called euglycemia.

The germinal idea of an artificial pancreas (AP) for T1DM patients was firstly envisioned 50 years ago in ${ }^{2}$. Nowadays, the main streams to control glycemia are PIDs and Model Predictive Control (MPC). This paper focuses on the latter approach. In the last decade, MPC received an increasing attention as an advanced control strategy to be implemented in an AP device 3 (4/56/67/89. In general, these formulations use discrete-time control actions and are based on a simplification of the model of T1DM patient presented in ${ }^{10}$ and its linearizations ${ }^{11 / 121331418}$. The most popular MPC formulation in AP is the zone MPC ${ }^{7 / 14}$ which, by means of a modified objective cost, considers an entire range of the controlled variable as the target. Recent improvements are the consideration of asymmetric cost functions and explicit velocity-penalties in the MPC formulation ${ }^{8}$.

From a pure control point of view, three main open problems rise from the aforementioned formulations: (i) most of the mathematical models available in the current literature are not consistent with the practical and popular Flexible Insulin Therapy (FIT), and thus provide wrong predictions when the prediction horizon is too large (because of the simplified stable model) ${ }^{15}$, 
(ii) even when they show to be stable, most of the controllers does not guarantee closed-loop stability (because they do not use any kind of stabilizing conditions, such as terminal costs and constraints, with the exception of ${ }^{355}$ that use an infinite predictions horizon but neither input nor state constraints are considered in the optimization problem) and (iii) most of the controllers do not properly exploit the stabilizable/controllable set of the closed-loop (i.e., the sets of states that can be stabilized) since they use a basal insulin infusion rate which is not computed/updated by the controller. Besides, from a technological perspective, the assumption of discrete (or continuous) control actions could not be realistic because insulin is administered by injections or insulin pumps that manipulate the duration of asynchronous pulses (even for the permanent basal infusion). These three drawbacks define three open problems which this paper is devoted to find a solution to. These three main contributions are summarized herein.

The solution to the first open problem consists in choosing a new prediction model which is consistent with real life medical practice as FIT. The first main message in this paper can be generalized to any model based control: it is expected that the performance is improved when using a long-term prediction, rather than inaccurate ones. More specifically, the main drawback of the classical prediction models is - as it was shown in ${ }^{1}$ - that they generate apparent equilibria during fasting periods, so that each value of blood glucose (BG) can be maintained constant thanks to a different insulin infusion rate. This stands in contradiction with real life and the well accepted Flexible Insulin Therapy, since patients display only one single insulin infusion rate, defined as the basal rate, which stabilizes the glycemia at any value as long as no meal is taken $\frac{16}{6}$. Opposite to what is done in the classical T1DM representations, the model developed in ${ }^{1}$ represents the patient realistically in a domain of validity, which mainly means that it has a critically stable equilibrium manifold (instead of a stable one). These equilibria correspond to an unique basal insulin injection level, and disturbances in BG (producing either hyper and hypoglycemia episodes) will not be self-regulated, if no additional actions (i.e., insulin boluses the case of hyperglycemia or insulin suspension the case of hypolycemia) are taken. In a similar vein as in ${ }^{1}$, this feature has been recently included in the model presented in ${ }^{[17}$ as well.

The second main contribution in this paper provides a solution to the second open problem by presenting a new stable Impulsive Zone Model Predictive Control (iZMPC) for T1DM patients. As announced above, in a first stage, a modification of the glucose-insulin model presented in ${ }^{1}$ is made to cope with medical practice and long-term predictions, while the good features of the classical control-relevant models are maintained. Having such a good long-term prediction of the insulin and carbohydrates (CHO) effect is crucial for an MPC to anticipate not only the blood glucose behavior, but also the eventual constraint activation ${ }^{18}$. In practical terms, the main benefit of this anticipatory behavior is to properly avoid hypoglycemic episodes and, thus, the need of additionally safety procedures that open the loop in dangerous situations. Then, in a second stage, the iZMPC is designed. By means of the use of artificial optimization variables, the controller guarantees recursive feasibility and stability of the closed-loop and shows an enlarged domain of attraction (in contrast to standard zone MPCs, with guaranteed stability) 1920121 . Furthermore, an impulsive scheme of the continuous-time original model is developed, which allows the controller to stabilize the BG by only injecting periodic insulin boluses of short duration, even for the basal rate (this impulsive basal rate, in addition, is automatically found by the controller according to the feedback). Though it seems to review some basic feature of the medical practice, this feature copes with the practical need of re-evaluation of the basal rate at different periods of the day.

The control scheme adopted in this work is depicted in Figure 1 and includes an update of the basal rate, which embodies a solution to the third open problem above. More precisely, the MPC controller computes the full insulin to be injected to the patient, i.e., the basal rate and the postprandial boluses, provided that the meal is (partially) announced.

Finally, the new Impulsive Zone MPC strategy is tested on several T1DM virtual patients. Long-term scenarios - including meals and patient parameter variations - are simulated. The results are satisfactory as smooth variable behaviours are obtained, while hypoglycemia episodes are avoided and hyperglycemia episodes are significantly reduced.

The outline of this paper is as follows. To account for the first contribution of the paper, Section 2 presents the new glucoseinsulin model and its physiological and dynamical description. This model is claimed to be instrumental to improve the prediction capacity of any model based control, including MPC. Then, to account for the second contribution, Section 3 introduces the impulsive Zone MPC. The way this controller, together with the proposed state observer, is implemented accounts for the third contribution of the paper. The MPC controller is described in detail in Section 3.1 whereas the observer is displayed in Section 4. Section 5 shows the results of the in silico trials, while the concluding remarks are given in Section 6 


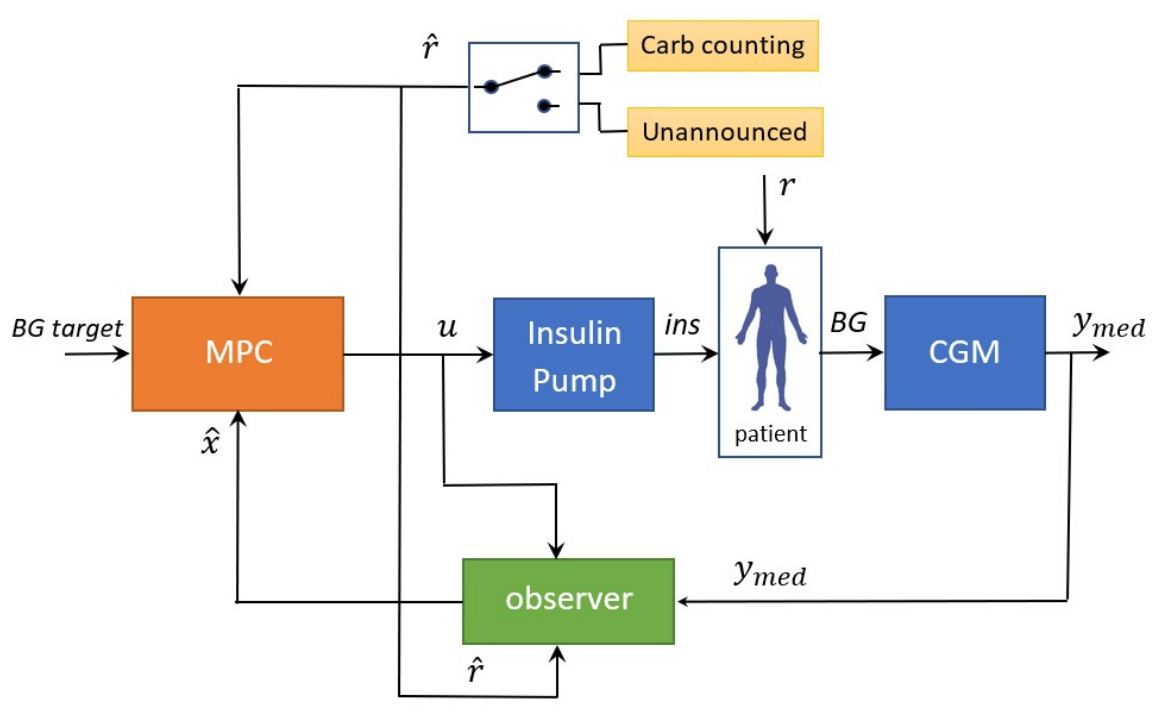

FIGURE 1 Control Scheme. The control objective is to maintain the blood glucose $(B G)$ in a safety range (BG target) by manipulating the insulin $(u)$ injected to the patient by means of a pump. The blood glucose is measured by means of a continuous glucose monitoring (CGM) while the meals intake $(r)$ is announced to the controller by means of $\hat{r}$. An observer is used to estimate the current model states $\hat{x}$, based on the CGM blood glucose measurement $y_{\text {med }}$, the insulin $u$ and the meal estimation $\hat{r}$.

\section{2 | GLUCOSE-INSULIN MODEL}

The glucose-insulin model is derived, based on the FIT paradigm, i.e. in the zone of interest one single constant basal rate has to be derived as well as other FIT parameters. The result of this approach is that the mathematical model has to be essentially linear with respect to insulin injection and to carbohydrates digestion. This linearity is, however, marginally distorted (when blood glucose concentration is close to zero) to cope with the positivity of the system. The model features better long-term predictions and is consistent with real life practice. It comprises three subsystems describing insulin absorption, meal absorption and glucose dynamics. This leads to 5 differential equations with 6 parameters and two inputs (exogenous insulin and meal), which represent the control and disturbance input, respectively.

\section{1 | Glucose Dynamics}

The glucose-insulin dynamics is dependent on four major factors: exogenous glucose intake, endogenous glucose production, exogenous insulin infusion and insulin-independent and insulin-dependent glucose utilization. This yields the following differential equation which is taken from ${ }^{22}$, for a type 1 diabetic patient:

$$
\frac{d G(t)}{d t}=-f_{1}(G(t))-f_{2}(G(t)) f_{3}\left(Q_{i}(t)\right)+f_{4}\left(Q_{i}(t)\right)+f_{5}(t),
$$

where $G$ is the blood glucose concentration (glycemia $[\mathrm{mg} / \mathrm{dl}]$ ) and $Q_{i}$ is the insulin delivery rate in plasma [U/min]. Function $f_{1}(\cdot)$ accounts for insulin-independent glucose utilization by the brain, $f_{2}(\cdot) f_{3}(\cdot)$, for insulin-dependent glucose utilization, $f_{4}(\cdot)$ for hepatic endogenous glucose production and $f_{5}(t)$ for the glycemia rise due to the digestion of carbohydrates $(\mathrm{CHO})$. The form of functions $f_{1}(\cdot)$ to $f_{4}(\cdot)$ were taken from ${ }^{22}$; Figure 2 below, illustrates their graphs. In particular, the functions $f_{1}$ and $f_{2}$ vanish when blood glucose concentration vanishes so that the model (1) fits to a so-called positive system.

It is desirable that the differential equation (1) copes with Flexible Insulin Therapy, at least from moderate hypoglycemia to moderate hyperglycemia, which is a range where FIT is assumed to be effective. The consequence is that in that range, the model (1) will be (almost) linear. Thus, define five glycemia zones as: severe hypoglycemia for $G<G_{s-h y p o}[\mathrm{mg} / \mathrm{dl}]$, hypoglycemia for $G_{\text {s-hypo }} \leq G<G_{\text {hypo }}$, euglycemia for $G_{\text {hypo }} \leq G<G_{\text {hyper }}$, hyperglycemia for $G_{\text {hyper }} \leq G<G_{\text {s-hyper }}$ and severe hyperglycemia for $G \geq G_{\text {s-hyper }}$ (see Figure 3 for a schematic plot). Some reasonable values for the BG limits are: $G_{s-h y p o} \approx 40$ [mg/dl], $G_{\text {hypo }} \approx 80[\mathrm{mg} / \mathrm{dl}], G_{\text {hyper }} \approx 140[\mathrm{mg} / \mathrm{dl}]$ and $G_{\text {s-hyper }} \approx 400[\mathrm{mg} / \mathrm{dl}]$. The range of interest for the linear model is the one 

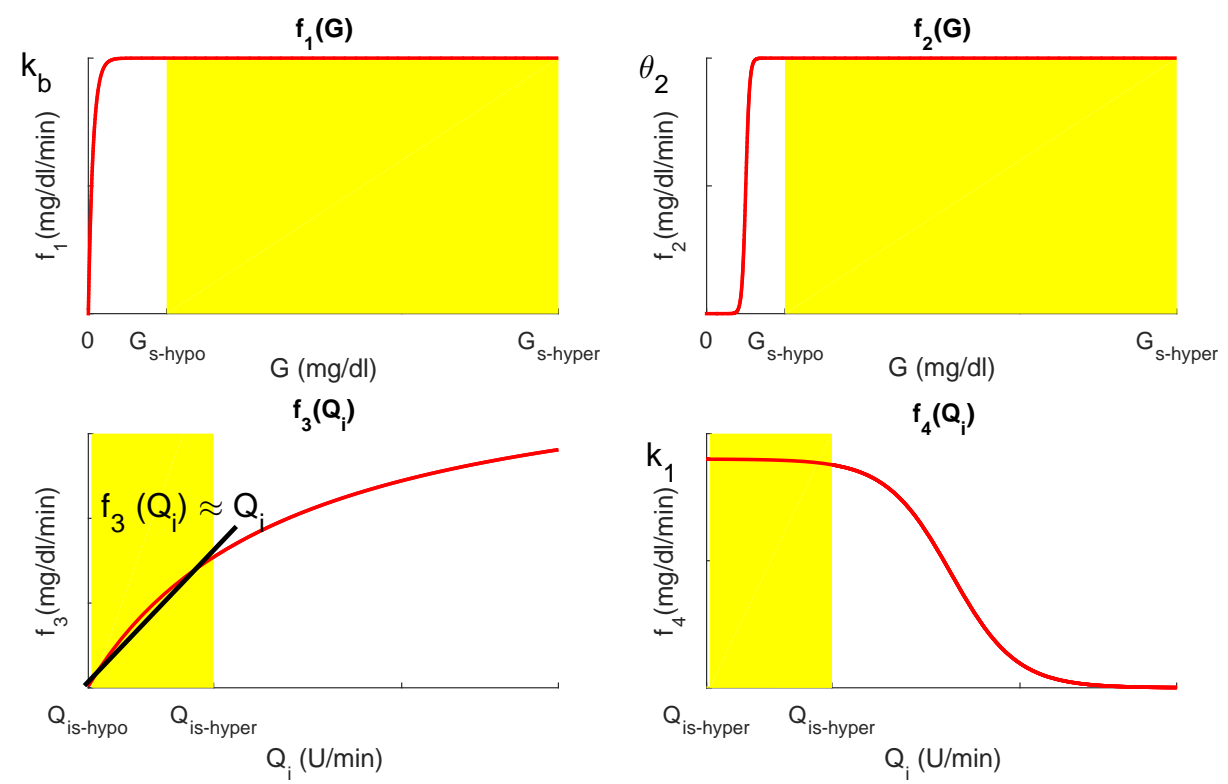

FIGURE 2 Illustrative plot of functions $f_{1}$ to $f_{4}$

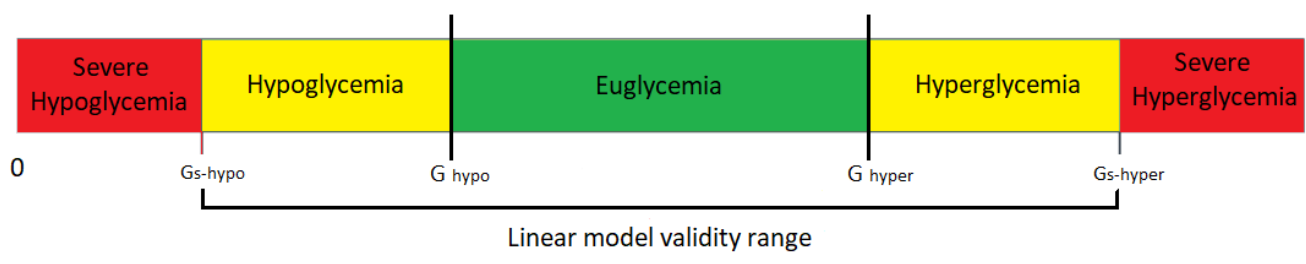

FIGURE 3 Linear fit validity glucose range

including hypo, eu and hyperglycemia, given that severe cases (both severe hypo and severe hyperglycemia) require specialized treatments (hospitalization with glucose or glucagon injection in case of hypoglycemia for instance) which is out of the scope of an automatic controller acting on insulin injection only.

According to the glycemia range of interest $G_{s-\text { hypo }} \leq G<G_{s-\text { hyper }}$, a corresponding insulin delivery rate range - in units per minute, [U/min] - can also be selected, $Q_{i_{s-h y p o}} \leq Q_{i}<Q_{i_{s-h y p e r}}$. Taking into account these two ranges: (i) function $f_{1}(\cdot)$ fits a constant function of $G$, i.e., $f_{1}(G) \approx k_{b}$, where $k_{b}$ represents the insulin-independent glucose utilization rate, specially by the brain; (ii) functions $f_{2}(\cdot)$ fits a constant function, i.e., $f_{2}(G) \approx \theta_{2}$, where $\theta_{2}$ represents the insulin sensitivity factor (ISF), (iii) function $f_{3}(\cdot)$ fits the identity function, i.e., $f_{3}\left(Q_{i}\right) \approx Q_{i}$, and (iv) $f_{4}(\cdot)$ also fits a constant function, i.e., $f_{4}\left(Q_{i}\right) \approx k_{1}$, where $k_{1}$ represents the liver endogenous glucose production. The yellow zones in Figure 2 show the model validity ranges of each linear function.

The so-obtained linear differential equation has, within the range of interest, the structure of the model in ${ }^{1}$, and reads as follows:

$$
\frac{d G(t)}{d t}=-k_{b}-\theta_{2} Q_{i}(t)+k_{1}+\theta_{4} Q_{d}(t),
$$

where $\theta_{4}$ is a parameter such that $\theta_{4} / \theta_{2}$ represents the insulin to carbohydrates ratio (ICR) and $Q_{d}(t)$ is the glucose delivery rate from the duodenum $[\mathrm{mg} / \mathrm{min}]$. As it can be seen, this equation has not a term depending linearly on the glucose $G$, which means that the derived model will be critically stable (integrating system). This kind of linear model properly represents the physiological equilibria in the desired glucose and insulin range of interest and, furthermore, the flexible insulin therapy (FIT) parameters are directly obtained from the model parameters, as it was established in ${ }^{1}$. 
Remark 1. Note that moderate-to-sever hyper and hypoglycemia scenarios are wrongly predicted by the linear approximation (2). In fact, for severe hyperglycemia the glucose stabilizes in a high value, instead of keeping growing, while for severe hypoglycemia the glucose stabilizes in a small positive value, instead of keeping decreasing. This behaviors are properly described by the general nonlinear equation (1) which features the expected properties of a so-called positive system.

\subsection{Insulin and Digestion Dynamics}

Following usual approaches in AP modeling $(\underline{117123})$ both, the insulin and digestion absorption subsystems, are assumed to be represented by linear dynamics, derived from compartmental models. The insulin absorption subsystem consists of two compartments: the subcutaneous and blood compartments. The resulting differential equations are given by 1 :

$$
\begin{aligned}
\frac{d Q_{i}(t)}{d t} & =-\frac{1}{\theta_{3}} Q_{i}(t)+\frac{1}{\theta_{3}} Q_{i_{s u b}}, \\
\frac{d Q_{i_{\text {sub }}}(t)}{d t} & =-\frac{1}{\theta_{3}} Q_{i_{\text {sub }}}(t)+\frac{1}{\theta_{3}} u(t)
\end{aligned}
$$

where $Q_{i_{\text {sub }}}$ stands for the insulin delivery rate in the subcutaneous compartment [U/min], $\theta_{3}$ is the time constant and $u(t)$ is the insulin infusion [U/min].

The digestion subsystem is also assumed to consist essentially of two compartments, which are in this case: the stomach and the duodenum. The resulting differential equations are given by:

$$
\begin{gathered}
\frac{d Q_{d}(t)}{d t}=-\frac{1}{\theta_{5}} Q_{d}(t)+\frac{1}{\theta_{5}} Q_{d_{s t o}}, \\
\frac{d Q_{d_{s t o}}(t)}{d t}=-\frac{1}{\theta_{5}} Q_{d_{s t o}}(t)+\frac{1}{\theta_{5}} r(t)
\end{gathered}
$$

where $Q_{d_{s t o}}$ stands for the glucose delivery rate from the stomach [mg/min], $\theta_{5}$ is the time constant and $r(t)$ is the amount of carbohydrate $\mathrm{CHO}$ in meals $[\mathrm{mg} / \mathrm{min}]$.

\subsection{Affine state space model}

Based on (2), (3) and (4), the following affine, continuous-time, state space model can be obtained:

$$
\begin{aligned}
\dot{x}(t) & =A x(t)+B_{u} u(t)+B_{r} r(t)+E, \quad x(0)=x_{0}, \\
y(t) & =C x(t),
\end{aligned}
$$

where $x(t)=\left[\begin{array}{lllll}x_{1}(t) & x_{2}(t) & x_{3}(t) & x_{4}(t) & x_{5}(t)\end{array}\right]^{\prime}$, with $x_{1}=G, x_{2}=Q_{i}, x_{3}=Q_{i_{s u b}}, x_{4}=Q_{d}$ and $x_{5}=Q_{d_{s t o}}$. The output $y(t)$ is given by the state component $x_{1}$, i.e., it represents the glycemia to be controlled. As before, $u(t)$ is the insulin infusion [U/min] and $r(t)$ is the amount of carbohydrate CHO in meals [mg/min]. $E$ is a constant term denoting the difference between the liver endogenous glucose production $\left(k_{1}\right)$ and the glucose absorption rate by the brain $\left(k_{b}\right)$.

The model matrices are given by:

$$
A=\left(\begin{array}{ccccc}
0 & -\theta_{2} & 0 & \theta_{4} & 0 \\
0 & -\frac{1}{\theta_{3}} & \frac{1}{\theta_{3}} & 0 & 0 \\
0 & 0 & -\frac{1}{\theta_{3}} & 0 & 0 \\
0 & 0 & 0 & -\frac{1}{\theta_{5}} & \frac{1}{\theta_{5}} \\
0 & 0 & 0 & 0 & -\frac{1}{\theta_{5}}
\end{array}\right), \quad B_{u}=\left(\begin{array}{c}
0 \\
0 \\
\frac{1}{\theta_{3}} \\
0 \\
0
\end{array}\right), \quad B_{r}=\left(\begin{array}{c}
0 \\
0 \\
0 \\
0 \\
\frac{1}{\theta_{5}}
\end{array}\right), \quad E=\left(\begin{array}{c}
\theta_{1} \\
0 \\
0 \\
0 \\
0
\end{array}\right), \quad C=\left(\begin{array}{lllll}
1 & 0 & 0 & 0 & 0
\end{array}\right),
$$

where $\theta_{1}=k_{1}-k_{b}$.

Constraints for both, states and inputs are considered, in such a way that $u \in \mathcal{V}, x \in \mathcal{X}$, where $\mathcal{V}$ and $\mathcal{X}$ are assumed to be polyhedrons in the positive orthant (i.e., every quantity is positive). Furthermore, it is also assumed that the amount of $\mathrm{CHO}$ is bounded by $0 \leq r \leq r_{\max }$, for a positive maximum $r_{\max }$.

Model (5 6] has a fixed parametric structure, in which the parameters represents physiological quantities. So, the individualization of the model according to each patient is performed by means of an identification (Gauss-Newton Identification Method ${ }^{24}$, in this case), which computes the set of parameters that minimizes the difference between historical input-output data (coming 
from the registered input and CGM data). The fact that parameters are physiological may (and in fact does) help in the identification process, given that it is easier to detect mistaken estimations. Although the model considered in this paper is essentially different to the others in literature, many other MPC-based approaches for AP consider this kind of individualization $25[5|26| 23 \mid 27$.

\subsection{Equilibrium and controllability characterization of the model}

An equilibrium couple of model (5) corresponding to fasting (i.e., couples $\left(u_{s}, x_{s}\right)$, such that $0=A x_{s}+B_{u} u_{s}+E$, considering $\left.r_{s}=0\right)$ is given by $x_{2, s}=\theta_{1} / \theta_{2}, x_{3, s}=\theta_{1} / \theta_{2}, x_{4, s}=0, x_{5}=0$ and any value of $x_{1, s}$, with $x_{s} \stackrel{\Delta}{=}\left[x_{1, s} x_{2, s} x_{3, s} x_{4, s} x_{5, s}\right]^{\prime}$. Furthermore, the corresponding equilibrium input $u_{s}$ is a fixed insulin value (denoted as basal insulin rate, $u_{b}$ ), given by $u_{s}=$ $\theta_{1} / \theta_{2}=u_{b}$. Note that the equilibrium glycemia level $y_{s}=x_{1, s}$ does not depend on $u_{s}$. Obviously, whatever its value is, the glycemia does depend on the past values of the insulin infusion $u(t)$ and meal intake $r(t)$.

These characteristics, that make the model essentially different from other models used in the literature (mainly the Bergman model ${ }^{10}$ and its simplified versions), permit to obtain long term predictions. This way, it is argued that every undesirable episode can be better anticipated and corrected. Regarding the controllability of model (5), it should be noted that only the first three states - accounting for the insulin-glycemia dynamics - are controllable, while the last two states from the digestion subsystem have their own dynamics, and they are obviously not affected by the insulin.

\section{5 | Impulsive inputs control scheme}

The main objective of this work - in contrast to other existing strategies - is to develop a MPC control strategy able to control the glycemia of a diabetic patient (described by model (5) by only using impulses (boluses) of insulin, without any continuous basal rate1

Under this framework - that we call impulsive input control scheme - we assume that the insulin infusion $u$ is injected into system (5) only by boluses applied at certain time instants denoted as $k T$, where $T$ is a fixed period, and $k \in \mathbb{N}$. This way, the system can be described by the following two equations:

$$
\begin{aligned}
\dot{x}(t) & =A x(t)+B_{r} r(t)+E, \quad x(0)=x_{0}, \quad t \neq k T, \\
x\left(k T^{+}\right) & =x(k T)+B_{u} u(k T), \quad k \in \mathbb{N},
\end{aligned}
$$

where $x\left(k T^{+}\right)$denotes the limit of $x(t)$ when $t$ approaches $k T$ from the right. This way, we have solutions containing discontinuities of first order (described by equation (8) and free responses affected only by the term associated to the meals $\left(B_{r} r(t)\right)$, between the discontinuities (described by equation (7)).

System (7)-(8) is a continuous-time system, but to implement the proposed MPC a discrete-time version (a sampled version, in fact) is needed. Based on the results presented in 21 , it is possible to have a sampled version of (7)-(8) that simultaneously accounts for both, the discontinuities and the free responses. The details and derivations of such a model are presented, for the sake of clarity, in the Appendix 8 What we need at this point to proceed with the MPC formulation is the discrete-time subsystem representing system (7)-(8) at the sampling times $k T$, which is given by:

$$
\begin{aligned}
x(k+1) & =A^{\bullet} x(k)+B_{u}^{\bullet} u(k)+B_{r}^{\bullet} r(k)+E, x(0)=x, \\
y(k) & =C x(k),
\end{aligned}
$$

where index $k$ represents the time $k T$, for $k \in \mathbb{N}$. The symbol $(\cdot)^{\bullet}$ denotes that the discrete-time system (9) describes the impulsive system (exactly) at the instants when the impulses occur 2 Given that system (7) has no formal equilibria (see Remark 3. in Appendix 8.1), a non-trivial extended equilibrium region must be defined for system 9 . The state and input equilibrium sets are denoted by $\mathcal{X}_{s}^{*}$ and $V_{s}^{*}$, respectively, and the details of their definition are given in Appendix 8.3

\footnotetext{
${ }^{1}$ Given that from a technological point of view, it could be desirable to have a minimal continuous flow to avoid catheter obstruction, a continuous small insulin flow can be considered.

${ }^{2}$ On the other hand, the symbol $(\cdot)^{\circ}$ is used (in the Appendix) to describe the impulsive system just an instant after the instant of the impulses.
} 


\section{3 | IMPULSIVE ZONE MPC}

The proposed MPC formulation has two distinctive mathematical characteristics: it is based on the impulsive input scheme - presented in subsection 2.5 and Appendix 8 - of the long-term affine model (5) and it makes use of artificial optimization variables to improve the feasibility properties $\left(\frac{1920)}{20}\right.$ and ensure stability.

The general MPC control objective is to keep the system in an equilibrium that maintains the glycemia $y(t)$ in a target zone inside the euglycemic range, by only manipulating the (positive and impulsive) insulin injection $u(t)$, while keeping the states $x_{1}(t), x_{2}(t)$ and $x_{3}(t)$ fulfilling the constraints. The target zone is defined as $\mathcal{Y}^{T a r} \stackrel{\Delta}{=}\left\{y \in \mathbb{R}: G_{\text {min }} \leq y \leq G_{\text {max }}\right\}$, with $G_{\text {min }} \geq G_{\text {hypo }}$ and $G_{\text {max }} \leq G_{\text {hyper }}$. As part of the control objectives, it should be noted that hypoglycemic episodes (when G is below the euglycemia zone) are much more dangerous, in the short term, than hyperglycemic episodes (when $G$ is above the euglycemia zone). Furthermore, the system under control is assumed to be perturbed by the effect of the $C H O$ in meals, $r(t)$ which is estimated only at the time it occurs - and by any other unknown disturbance producing a variation of BG (subject stress and exercise, dawn phenomenon, etc.).

\section{1 | Controller Formulation}

Let us define the impulsive state target set, $\mathcal{X}_{s}^{\bullet T a r} \subseteq \mathcal{X}_{s}^{\bullet}$, such that $C \mathcal{X}_{s}^{\bullet T a r}=\mathcal{Y}^{\text {Tar }}$, and the impulsive input target set as $\mathcal{V}_{s}^{\bullet T a r}=$ $\left\{u_{b}^{\cdot}\right\}$, with $\left\{u_{b}^{\cdot}\right\}$ being the impulsive basal rate of model 9 (see Appendix 8.3). Following the ideas presented in 192021 , the cost of the optimization problem that the proposed MPC solves on-line reads

$$
V_{N}\left(\hat{x}, \hat{r}, \mathcal{X}_{s}^{\cdot T a r}, \mathcal{V}_{s}^{\bullet T a r} ; \mathbf{u}, u_{a}, x_{a}\right) \stackrel{\Delta}{=} V_{d y n}\left(\hat{x}, \hat{r} ; \mathbf{u}, u_{a}, x_{a}\right)+V_{s t a}\left(\mathcal{X}_{s}^{\bullet T a r}, \mathcal{V}_{s}^{\bullet T a r} ; u_{a}, x_{a}\right),
$$

where

$$
V_{d y n}\left(\hat{x}, \hat{r} ; \mathbf{u}, u_{a}, x_{a}\right) \stackrel{\Delta}{=} \sum_{j=0}^{N-1}\left\|x(j)-x_{a}\right\|_{Q}^{2}+\left\|u(j)-u_{a}\right\|_{R}^{2},
$$

with $Q>0$ and $R>0$, is a term devoted to drive the system to the artificial equilibrium variable given by the artificial pair $\left(u_{a}, x_{a}\right) \in \mathcal{V}_{s}^{\bullet} \times \mathcal{X}_{s}^{*}$, and

$$
V_{s t a}\left(\mathcal{X}_{s}^{\bullet T a r}, \mathcal{V}_{s}^{T a r} ; u_{a}, x_{a}\right) \stackrel{\Delta}{=} p\left(\operatorname{dist}_{C \mathcal{X}_{s}^{\cdot T a r}}\left(C x_{a}\right)+\operatorname{dist}_{\bigvee_{s}^{\bullet T a r}}\left(u_{a}\right)\right)
$$

with $p>0$ and $\operatorname{dist}_{\mathcal{A}}(a)$ representing the distance from the point $a$ to the set $\mathcal{A}$, is a terminal cost devoted to drive $C x_{a}$ to the sets $C \mathcal{X}_{s}^{\cdot T a r}$ and $u_{a}$ to $V_{s}^{\cdot T a r}$, respectively. $\hat{x}$ and $\hat{r}$ represent the current estimation of the state and meal, respectively.

Note that in the latter cost, the current estimated state $\hat{x}$, the current estimated disturbance $\hat{r}$, and the target sets $\mathcal{X}_{s}^{\bullet T a r}$ and $V_{s}^{\cdot} \cdot \operatorname{Tar}$ are optimization parameters, while $\mathbf{u}=\{u(0), u(1), \cdots, u(N-1)\}, u_{a}$ and $x_{a}$ are the optimization variables $(N$ being the control horizon).

Remark 2. The latter cost is a zone-symmetric quadratic cost, that penalizes the distance to the desired target set (zone), in a symmetric form. Many alternatives can be analyzed to improve this cost, in order to harder penalize the hypoglycemia episodes ${ }^{28}$, according to a particular metric.

The optimization problem to be solved at time $k$ by the MPC is given by

$$
\begin{aligned}
& P_{M P C}\left(\hat{x}, \hat{r}, \mathcal{X}_{s}^{\bullet T a r}, \mathcal{V}_{s}^{\cdot T a r}\right): \\
& \min _{\mathbf{u}, u_{a}, x_{a}} V_{N}\left(\hat{x}, \hat{r}, \mathcal{X}_{s}^{\bullet T a r}, \mathcal{V}_{s}^{\bullet T a r} ; \mathbf{u}, u_{a}, x_{a}\right) \\
& \text { s.t. } \\
& x(0)=\hat{x}, r(0)=\hat{r}
\end{aligned}
$$

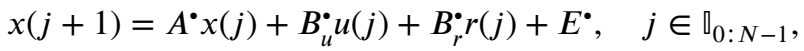

$$
\begin{aligned}
& r(j)=0, \quad j \in \mathbb{\square}_{1: N-1} \text {, } \\
& \tilde{C} x(j) \in \tilde{C} \mathcal{X}, u(j) \in \mathcal{V}, \quad j \in \mathbb{0}_{0: N-1} \text {, } \\
& \tilde{C} x(N)=\tilde{C} x_{a} \text {, } \\
& \tilde{C} x_{a}=\tilde{C}\left(A^{\bullet} x_{a}+B_{u}^{\bullet} u_{a}+E^{\bullet}\right) \text {. }
\end{aligned}
$$


Given that only the current meal is estimated (i.e., no future information is considered), $r$ is only used for the first prediction step, if available. Furthermore, given that only the first three states are controllable, and constraints satisfaction for states $x_{4}$ and $x_{5}$ cannot be required, matrix $\tilde{C}=\left[\begin{array}{ll}I_{3} & 0_{3 \times 2}\end{array}\right]$ is defined to extract the states $x_{1}, x_{2}$ and $x_{3}$ from the complete vector state $x$.

Constraint $\tilde{C} x(N)=\tilde{C} x_{a}$ is the terminal constraint that forces the terminal controllable state - at the end of control horizon $N$ - to reach the artificial equilibrium state $\tilde{C} x_{a}$. Furthermore, the last constraint forces the artificial variable pair $\left(u_{a}, x_{a}\right)$ to be in $\mathcal{X}_{s}^{\cdot} \times \mathcal{V}_{s}^{\cdot}$. These two constraints force the state at the end of the horizon to be any feasible equilibrium state (and not a specific one in the target set), corresponding to the fasting scenario $(r \equiv 0)$. This flexible constraints, which gives to the problem extra degrees of freedom, are necessary to ensure the closed-loop stability, as it is shown in 21 .

Once the Problem $P_{M P C}\left(x, r, \mathcal{X}_{s}^{\cdot T a r}, \mathcal{V}_{s}^{\cdot T a r}\right)$ is solved, the (optimal) solution is denoted as $\left(\mathbf{u}^{0}, u_{a}^{0}, x_{a}^{0}\right)$, while the optimal cost

function is given by $V_{N}^{0}\left(x, r, \mathcal{X}_{s}^{\cdot T a r}, \mathcal{V}_{s}^{\cdot T a r}\right) \stackrel{\Delta}{=} V_{N}\left(x, r, \mathcal{X}_{s}^{\cdot T a r}, \mathcal{V}_{s}^{\cdot T a r} ; \mathbf{u}^{0}, u_{a}^{0}, x_{a}^{0}\right)$. The control law, derived from the application of a receding horizon control policy (RHC), is given by $\kappa_{M P C}(x)=\kappa_{M P C}\left(x ; r, \mathcal{X}_{s}^{\cdot T a r}, \vartheta_{s}^{T a r}\right)=u^{0}(0 ; x)$, where $u^{0}(0 ; x)$ is the first element of the solution sequence $\mathbf{u}^{0}(x)$. The domain of attraction of the closed loop, $\mathcal{X}_{N}^{*}$, is given by the controllable set in $N$ steps to the entire equilibrium set $\mathcal{X}_{s}^{*}$ (in contrast to classical stable MPC formulations, for which the domain of attraction is given by the controllable set in $N$ steps to the equilibrium objective zone).

As it was done with the model parameters, the MPC tuning parameters should be individualized for each patient. Unfortunately, as it is known from the MPC literature, there is no a formal general procedure to do that. In the simulation section 5 some general tuning steps related to the difference between the insulin and CHO subsystem gains and the corresponding time constants, will be presented.

The next property formalizes the closed-loop stability of the MPC.

Property 1. The equilibrium set $\mathcal{X}_{s}^{\cdot T a r}$ is asymptotically stable under the closed-loop system $x(k+1)=A^{\bullet} x(k)+$ $B_{u}^{*} \kappa_{M P C}(x(k))+E^{\bullet}$. Furthermore, given $x(k) \in \mathcal{X}_{N}^{*}$, the optimization problem $P_{M P C}(\cdot)$ is feasible for $x(k+1)$, and so, for every future state of the closed-loop.

Proof: The proof of stability and recursive feasibility follows the same steps of the proof of Theorem 3 , in 21 .

\section{2 | Closed-loop benefits}

The new MPC formulation herein enjoys several properties that are to be mentioned.

(i) Due to the proper use of artificial variables, the MPC not only has an enlarged domain of attraction, but also ensures constraint satisfaction and stability ${ }^{212930}$, for moderate-to-large disturbances and model-plant mismatches. Furthermore, the latter properties are achieved by only solving a sequence of quadratic programming problems $(\mathrm{QP})$, which means that no computational complexity is added.

(ii) The use of a long-term model for predictions permits to better exploit the anticipative benefits of the strategy. The early prediction of possible hypoglycemic episodes that the insulin will not be able to correct by means of feasible actions (because of its positivity) is the key point to properly avoid them.

(iii) The use of impulses for both, basal and bolus infusions, improves the general controllability of the closed loop. (i.e., the general ability to reach any desired state). It also contributes to better avoid hypoglycemic episodes, since no permanent insulin infusion is used. The use of impulses also improves the pharmacokinetics (due to a potentially higher insulin absorption from the subcutaneous infusion site), the performance of insulin pumps and, furthermore, injections could also be used to implement the control.

(iv) The MPC computes by itself the impulsive insulin basal rate (there is no need of an off-line computation), which permits to easily cope with the frequent variations of its value. From the MPC point of view, even when there is model-plant mismatch, the basal rate is just the equilibrium of the closed loop.

(v) The effect of the so-called insulin on board $\left(I_{o b}\right)$ is automatically accounted, in the predictions, by the long-term model. This means that its value does not need, in principle, to be computed from some external identification algorithm.

(vi) Under a meal-announcement scenario, the postprandial insulin boluses are computed (optimally) by the MPC, according to the BG predictions. This means that not only the postprandial insulin bolus is optimal (according to the long-term model, which includes a meal subsystem), but also that all possible (moderate) variations of the system are taken into account, by means of the state feedback. 


\section{4 | STATE ESTIMATION}

The state estimation is crucial to account for a realistic closed-loop performance in the artificial pancreas problem. In this work, a Kalman filter based on the following extended model is used 31 :

$$
\begin{aligned}
{\left[\begin{array}{l}
x(k) \\
d(k)
\end{array}\right] } & =\underbrace{\left[\begin{array}{cc}
A^{\bullet} & M_{e} \\
0 & 1
\end{array}\right]}_{A^{\text {ext }}}\left[\begin{array}{c}
x(k-1) \\
d(k-1)
\end{array}\right]+\underbrace{\left[\begin{array}{cc}
B_{u}^{*} B_{r}^{*} & E^{\bullet} \\
0
\end{array}\right]}_{B^{\text {ext }}}\left[\begin{array}{c}
u(k-1) \\
r(k-1) \\
1
\end{array}\right]+G_{w} w(k-1) \\
y(k) & =\left[\begin{array}{ll}
C & 0
\end{array}\right]\left[\begin{array}{l}
x(k) \\
d(k)
\end{array}\right]+v(k)
\end{aligned}
$$

where $M_{e}=\left[\begin{array}{lllll}1 & 0 & 0 & 1 & 1\end{array}\right]^{T}, G_{w}=\left[\begin{array}{llllll}0 & 0 & 0 & 0 & 0 & 1\end{array}\right]^{T}, w(k) \in \mathbb{R}$ is assumed to be a zero-mean Gaussian process noise, with variance $Q^{\text {ext }}$, and $v(k) \in \mathbb{R}$ is assumed to be a zero-mean Gaussian output noise with variance $R^{\text {ext }}$. The value of $R$ is computed according to variance of the Continuous Glucose Monitor (CGM) (for instance, Dexcom G5 Mobile CGM,, 32 ), while the value of $Q$ is used as a tuning parameter for the performance of the estimation. Roughly speaking, a large $Q^{\text {ext }} / R^{\text {ext }}$ ratio is selected when the measurement is confident, while a small $Q^{\text {ext }} / R^{\text {ext }}$ ratio is selected when the measurement noise is high, and the model is accurate.

The Kalman filter is derived from the following recursion:

Prediction:

$$
\begin{aligned}
\hat{x}^{-}(j) & =A^{\text {ext }} \hat{x}(j-1)+B^{e x t}\left[\begin{array}{c}
u(j) \\
\hat{r}(j) \\
1
\end{array}\right], \\
P_{j}^{-} & =A^{\text {ext }} P_{j-1} A^{\text {ext } T}+G_{w} Q^{\text {ext }} G_{w}^{T}
\end{aligned}
$$

Update:

$$
\begin{aligned}
K_{j} & =P_{j}^{-} C^{T}\left(C P_{j}^{-} C^{T}+R^{e x t}\right)^{-1} \\
\hat{x}(j) & =\hat{x}^{-}(j)+K_{j}\left(y_{m e d}(j)-C \hat{x}^{-}(j)\right) \\
P_{j} & =\left(I_{n x}-K_{j} C\right) P_{j}^{-}
\end{aligned}
$$

where $P_{0}=I_{6}, y_{\text {med }}$ is the measured glycemia and $\hat{x}$ and $\hat{r}$ are the estimated state and disturbance (meal), respectively.

As described in Sections 2.1 to 2.4 the parameters and states of the prediction model are directly related to physiological variables. The states $x_{2}$ and $x_{3}$ describe the insulin pharmacokinetics, and an acceptable estimation of such states permits to know (approximately) the insulin that is still in the body (known as insulin on board $I_{o b}$ ). In principle, and from the theoretic contributions above, this estimation minimizes the need of additional safety procedures to limit the $I_{o b}$, and consequently the risk of hypoglycemia. Nevertheless, when it comes to clinical trials, then any control algorithm requires a universal safety supervisor layer to guarantee the safety of patients. Furthermore, in scenarios of unannounced or partially announced meals, the estimation of states $x_{4}$ and $x_{5}$ (representing the dynamic/absorption of the CHO after a meal), becomes crucial. A further benefit of model (5), coming form the fact that the parameter and states are physiological quantities, is that both, the identification and estimation procedure can be assessed, if some measurement of these quantities are available.

\section{5 | SIMULATION RESULTS}

The developed impulsive ZMPC controller is tested to regulate glycemia to a target zone (defined by $\mathcal{Y}^{\text {Tar }}$ ), based on the T1DM patient model described above, and subject to the corresponding constraints. The general control scheme adopted for the simulation is the one shown in Figure 1 Two general cases are considered: (i) model-plant mismatch, with fixed parameter and temporary disturbances. In this case, the plant is the continuous-time virtual patient but with parameters significantly modified using a fixed percentage, while the original model is used to generate the prediction. Furthermore, the dawn phenomenon, physical exercise of the patient and a catheter change are simulated as the temporary disturbances. The second case (ii) uses the UVA/Padova simulator as the plant, and several scenarios are selected to show how the controller is able to achieve a good performance even when the plant has a different nature from the virtual patient prediction model. 
For the two cases, the simulation time is settled to $48 \mathrm{~h}$. This duration of the simulation is longer than the one in other works ${ }^{76 / 33}$, since the linear model which is used is able to reproduce accurately the behavior for long-time intervals. The state and input constraints are given by $\mathcal{X}=\left\{x:\left[\begin{array}{llll}0 & 0 & 0 & \text { none none }\end{array}\right]^{T} \leq x \leq\left[\begin{array}{lllll}500 & 10 & 10 & \text { none none }\end{array}\right]^{T}\right\}$, where none denotes that there is no constraint on the non-controllable states, and $V=\{u: 0 \leq u \leq 30\}$, respectively. The time period was selected to be $T=15$ (min) to stress the fact that sporadic insulin injections are able to produce a good BG performances. Furthermore, many clinical trials work with this period. In general, this period should be selected according to the speed of the insulin and food effects. This must be done because the controller can inject insulin only at times $k T, k \in \mathbb{N}$. If a disturbance affects the system at time $t=k T+\Delta T$, for instance, with $\Delta T<<T$, then the controller action is delayed for almost $T$ minutes before beginning to compensate it.

The gain $(G)$ and settling time ( $S T)$ of the BG response of each patient to the insulin injection ${ }_{u}{ }_{u}$ ) and food intake $\left.{ }_{r}\right)$ can be defined as $G_{u}=\theta_{2}, S T_{u} \approx 6 \theta_{3}, G_{r}=\theta_{4}$ and $S T_{r} \approx 6 \theta_{5}$, respectively. Clearly, for patients with larger values of $G_{u}$ and $S T_{u}$ in comparison with $G_{r}$ and $S T_{r}$, the meal intake disturbances can be quickly compensated by the insulin injection, reducing the potential time exceeding the safety upper limit $G_{\max }$, and in consequence, reducing potential hyperglycemic episodes. However, in this case (specially for large values of $G_{u}$ ), it is also easy to cause hypoglycemic episodes. In a similar way, for larger values of $G_{r}$ and $S T_{r}$ in comparison with $G_{u}$ and $S T_{u}$, hypoglycemic episodes are quite easy to be avoided, while hyperglycemic episodes will be slowly compensated ${ }^{3}$

An important fact of the artificial pancreas problem is that the most dangerous short-term closed-loop hypoglycemic episodes are exclusively produced by (a wrong action of) the controller, since a Type 1 diabetic patient does not experience this kind of episodes naturally. This is a quite different paradigm for an MPC controller, since it implies that in each time that an insulin injection is decided, it should be carefully computed to avoid, after the meal effect rejection, an undesirable drop of the blood glucose. More precisely, the insulin long-term effect must be carefully considered to prevent a future input constraint activation at zero.

Based on the latter analysis, the tuning MPC parameters can be selected to have a more conservative controller in the case of high insulin sensitivity and vice-versa. In this paper, the parameters of the MPC that will be selected according to the patient parameters are the control/prediction horizon $N$, the penalties $Q$ and $R$, and the limits of the output zone, $G_{\min }$ and $G_{\max }$, which constitute the output target $\mathcal{Y}^{T a r}=\left\{y \in \mathbb{R}: G_{\min } \leq y \leq G_{\max }\right\} . N$ is selected to be large enough to account for the entire insulin effect, given that overdoses are hard to compensate, because of the positiveness of the control action. The selected formula is $N \approx \frac{S T_{u} \times 60}{T}$, which must be understood just as a practical rule. Note that the use of larger horizons $N$ causes a higher computational effort. The target limits are selected such that $80 \leq G_{\min } \leq 100$ and $100 \leq G_{\max } \leq 140$. Note that the proposed control strategy makes no distinction between BG values inside the target zone, and so, it will maintain basal rate delivery as soon as the BG enters $\mathcal{Y}^{T a r}$, even if it is placed at the boundary of this set.

\section{1 | Case 1: Model-plant mismatch, with fixed parameters}

According to ${ }^{1}$, eight virtual patients are considered (labeled as PV1, PV2, PV3, PV5, PV6, PV10, PV11 and PV15), whose model parameters were obtained and validated from real patient data provided by the Nantes University Hospital and Rennes University Hospital in France, through an ad hoc anonymized collection. The main model parameters and the patient-dependent MPC parameters are shown in Table 1 The meal scenario is selected to simulate three meals in a day (breakfast, lunch and dinner), with the following amounts of $\mathrm{CHO}: 50 \mathrm{~g}$ at 7:00, $80 \mathrm{~g}$ at 12:00 and $60 \mathrm{~g}$ at 21:00. The relationship between the model and the plant parameters was selected in such a way that hypoglycemic episodes are more likely to occur (insulin effects are underestimated while $\mathrm{CHO}$ effects are overestimated), and they are shown in Table 2 . Additionally, to further stress the controller, both the dawn phenomenon and the patient physical exercises are considered. The dawn phenomenon is produced by a hormone release that stimulates the liver endogenous glucose production, but also diminishes the insulin sensitivity. So, to simulate this phenomenon, parameter $\theta_{2, \text { real }}$ (ISF) is reduced to $60 \%$ of its actual value, between 4:00 and 8:00 hs. To cope with the physical exercises, parameter $\theta_{2, \text { real }}$ is increased to $130 \%$ of its actual value, between 16:00 and 20:00. To represent the effect of a catheter change, parameter $\theta_{3, \text { real }}$ is reduced to $90 \%$ of its actual value in the second day of the simulation. Finally, given that both, the controller and the observer use a synchronous announcement of the meal to predict the glycemia or estimate the states, a mismatch is also included in this value. For breakfast and dinner, the $\mathrm{CHO}$ in meals that the controller and observer use is $85 \%$ of the actual value, while for the lunch it is $115 \%$.

${ }^{3}$ All these observations are true as long as the model properly matches the virtual patient. 
TABLE 1 Model and MPC parameters

\begin{tabular}{|c|c|c|c|c|c|c|c|}
\hline Pat. & $G_{u}$ & $S T_{u}(h)$ & $G_{r}$ & $S T_{r}(h)$ & $N$ & $(Q, R, p)$ & {$\left[G_{\operatorname{mim}}-G_{\max }\right]$} \\
\hline \hline PV1 & 69.68 & 6.51 & 4.10 & 2.40 & 31 & $\left(0.1 ; 1 e^{3}, 1 e^{5}\right)$ & {$[110-135]$} \\
\hline PV2 & 14.94 & 13.62 & 2.88 & 16.98 & 64 & $\left(0.1 ; 1 e^{3}, 1 e^{5}\right)$ & {$[120-140]$} \\
\hline PV3 & 18.30 & 8.90 & 2.07 & 4.37 & 42 & $\left(1.5 ; 1 e^{3}, 1 e^{5}\right)$ & {$[110-130]$} \\
\hline PV5 & 48.45 & 6.24 & 6.75 & 4.32 & 30 & $\left(0.1 ; 1 e^{3}, 1 e^{5}\right)$ & {$[110-130]$} \\
\hline PV6 & 16.08 & 14.98 & 2.07 & 3.82 & 70 & $\left(0.5 ; 1 e^{3}, 1 e^{5}\right)$ & {$[110-130]$} \\
\hline PV10 & 10.94 & 6.27 & 2.76 & 3.39 & 30 & $\left(1.75 ; 1 e^{3}, 1 e^{5}\right)$ & {$[110-130]$} \\
\hline PV11 & 27.30 & 5.89 & 4.61 & 6.74 & 28 & $\left(1 ; 1 e^{3}, 1 e^{5}\right)$ & {$[110-135]$} \\
\hline PV15 & 7.83 & 12.85 & 1.24 & 1.75 & 60 & $\left(15 ; 1 e^{3}, 1 e^{5}\right)$ & {$[110-130]$} \\
\hline
\end{tabular}

TABLE 2 Model-plant mismatch for Case 1

\begin{tabular}{|c|c|}
\hline$\theta_{2, \text { model }}=\theta_{2, \text { real }} / 1.3$ & the insulin effect is underestimated \\
\hline$\theta_{3, \text { model }}=\theta_{3, \text { real }} / 1.3$ & the insulin on board is underestimated \\
\hline$\theta_{4, \text { model }}=\theta_{4, \text { real }} / 0.7$ & the CHO-in-meals effect is overestimated \\
\hline$\theta_{1, \text { model }}=\theta_{1, \text { real }} / 0.7$ & the basal rate is overestimated \\
\hline
\end{tabular}

The states are estimated by means of a Kalman filter, based on an extended model considering an output disturbance. It is assumed that the BG measurement and the insulin infusion are available (known), while the current $\mathrm{CHO}$ in meals is only partially known, depending on the simulated scenario.

The BG and $\mathrm{CHO}$ in meals time evolutions are shown in Figure 4 for the eight simulated patients. The display includes the BG mean, the BG mean \pm a standard deviation and the envelope for all curves. As it can be seen, even with the large dispersion of the model parameters, the controller is able to avoid hyperglycemic episodes, in the long term, and hypoglycemic episodes, in the short term. Furthermore, it must be noted that this performance is achieved by only injecting insulin by impulses, in periods of 15 minutes.

On one hand, Figure 5 shows the BG and CHO evolution for a particular patient, PV15. The evolution of the auxiliary state variable $x_{a, 1}$ is shown in red and corresponds to the BG. As expected, this auxiliary variable remains most of time inside the target zone $\mathcal{Y}^{T a r}=\{G: 110 \leq G \leq 130\}$, because of a high terminal penalty $p$, so that the BG evolution is strongly penalized when it is outside the target region. Furthermore, when a strong disturbance affects the system, this auxiliary variable leaves the zone if necessary, to allow the controller to reject the disturbance (see the $x_{a, 1}$ evolution at times 13 and 37). Figure 6 , on the other hand, shows the evolution of the input injections. This Figure also includes the artificial input variable $u_{a}$, displayed in red, which plays a similar role than $x_{a}$ and the evolution of the third state of the virtual patient, which denotes the insulin in plasma.

\section{2 | Case 2: UVA/Padova simulations}

The UVA/Padova type 1 diabetic patient simulator ${ }^{\sqrt{34}}$ is commercially available and the only one which was approved by the US Food and Drug Administration for pre-clinical trials.

In this subsection, some of the benefits of the control structure in Section 3 are demonstrated on the Academic version v3.2, 2013, of the UVA/Padova simulator. To stress the controller, a period of $T=15$ minutes is selected.

In contrast to what was made in the previous subsection, pulse inputs of one minute of duration are used here instead of impulsive inputs for a proper simulation in the UVA/Padova virtual patien 4 To properly represent model (5) under the pulse scheme, some modifications should be made on the underlying discrete-time subsystems, as it is detailed in Subsection 8.4 of the Appendix.

The pattern of the simulated scenario is the same as the one in the previous subsection including breakfast, lunch and dinner, over two days. However, some modifications were made in both the amount and the schedule of each meal. Furthermore, a snack

${ }^{4}$ The UVA/Padova virtual patients are treated by insulin injections of at least one per minute. Remark that one minute remains a very short time in comparison with $T$. 


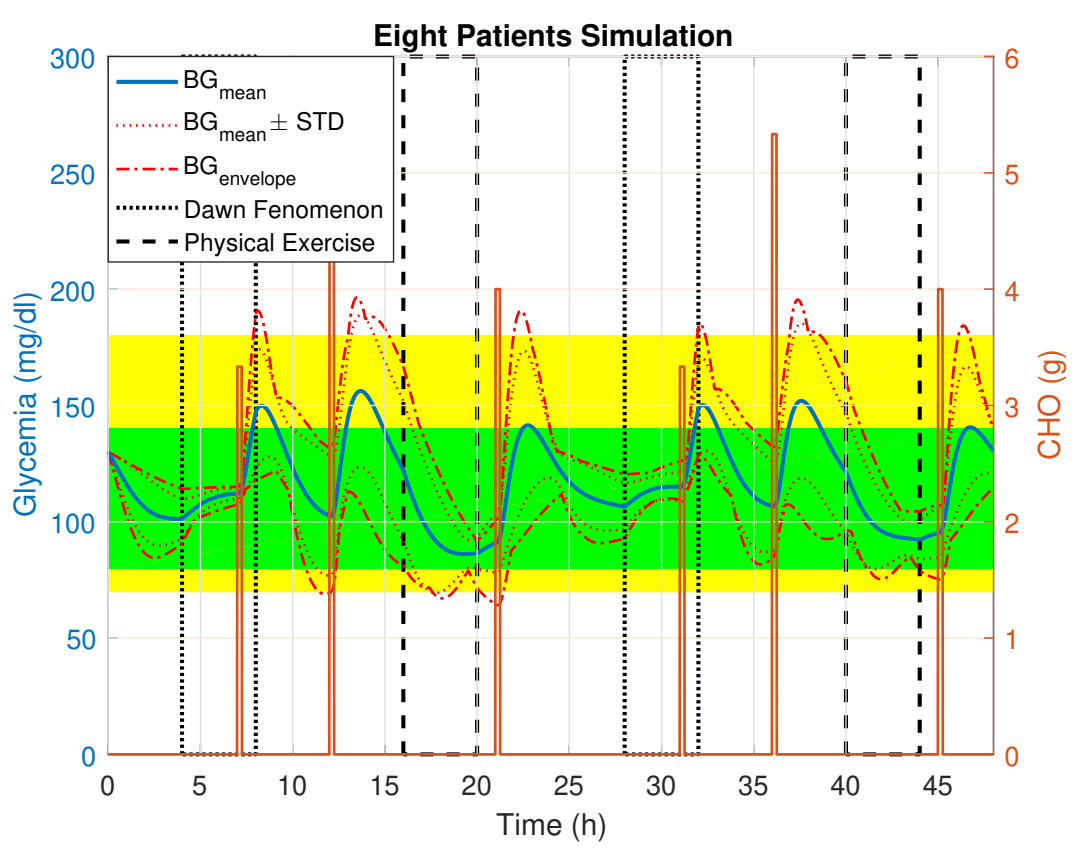

FIGURE 4 Eight patients BG (blue line) and CHO in meals (orange line) evolutions. The yellow glycemic range is given by $70-180 \mathrm{mg} / \mathrm{dl}$, while the green one is given by the euglycemia range $80-140 \mathrm{mg} / \mathrm{dl}$. Both, the dawn phenomenon and the exercises disturbance are indicated in the plot.

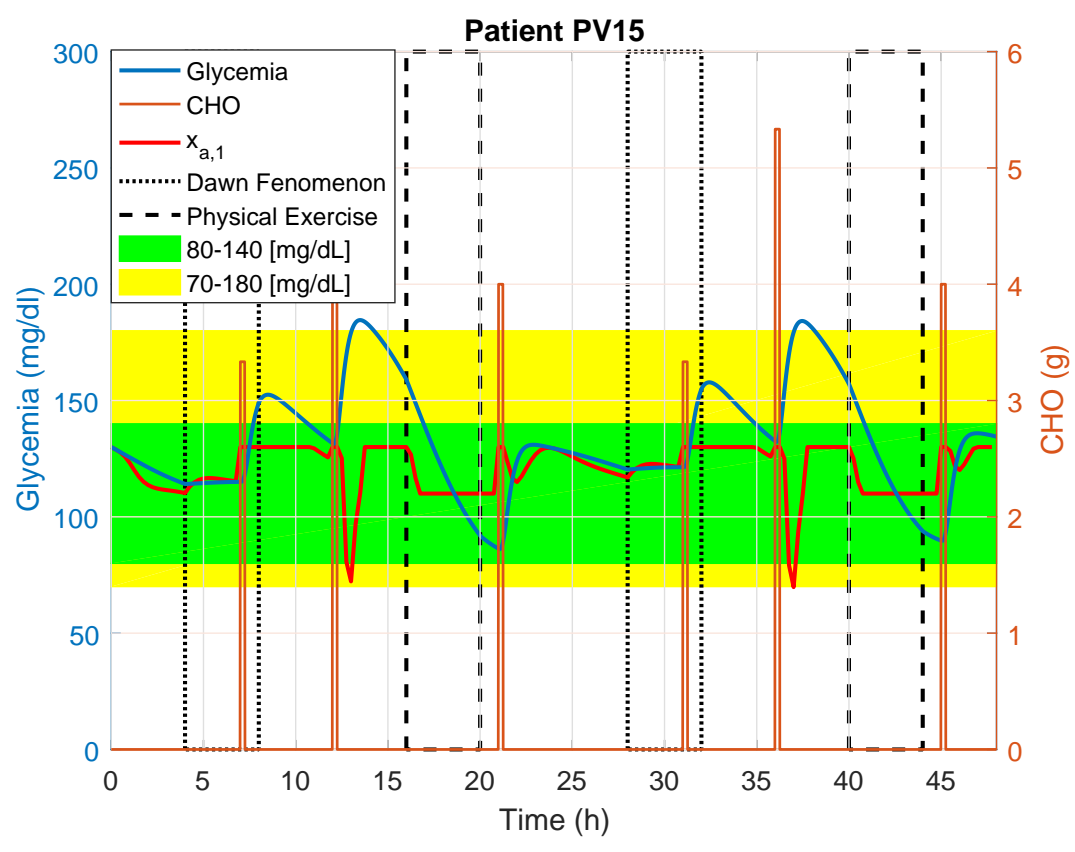

FIGURE 5 PV15 patient. Glycemia evolution (blue line) and CHO in meals (orange line). The yellow glycemic range is given by $70-180 \mathrm{mg} / \mathrm{dl}$, while the green one is given by the euglycemia range $80-140 \mathrm{mg} / \mathrm{dl}$. Both, the dawn phenomenon and the exercises disturbance are indicated in the plot.

is included between lunch and dinner. The meals time and amount are: $50 \mathrm{~g}$ at $7: 00,80 \mathrm{~g}$ at $13: 00,25 \mathrm{~g}$ at $17: 00,60 \mathrm{~g}$ at $20: 00$ for the first day, and $55 \mathrm{~g}$ at $6: 00,80 \mathrm{~g}$ at $14: 00,30 \mathrm{~g}$ at $18: 00,55 \mathrm{~g}$ at $21: 00$ for the second day. The selected 


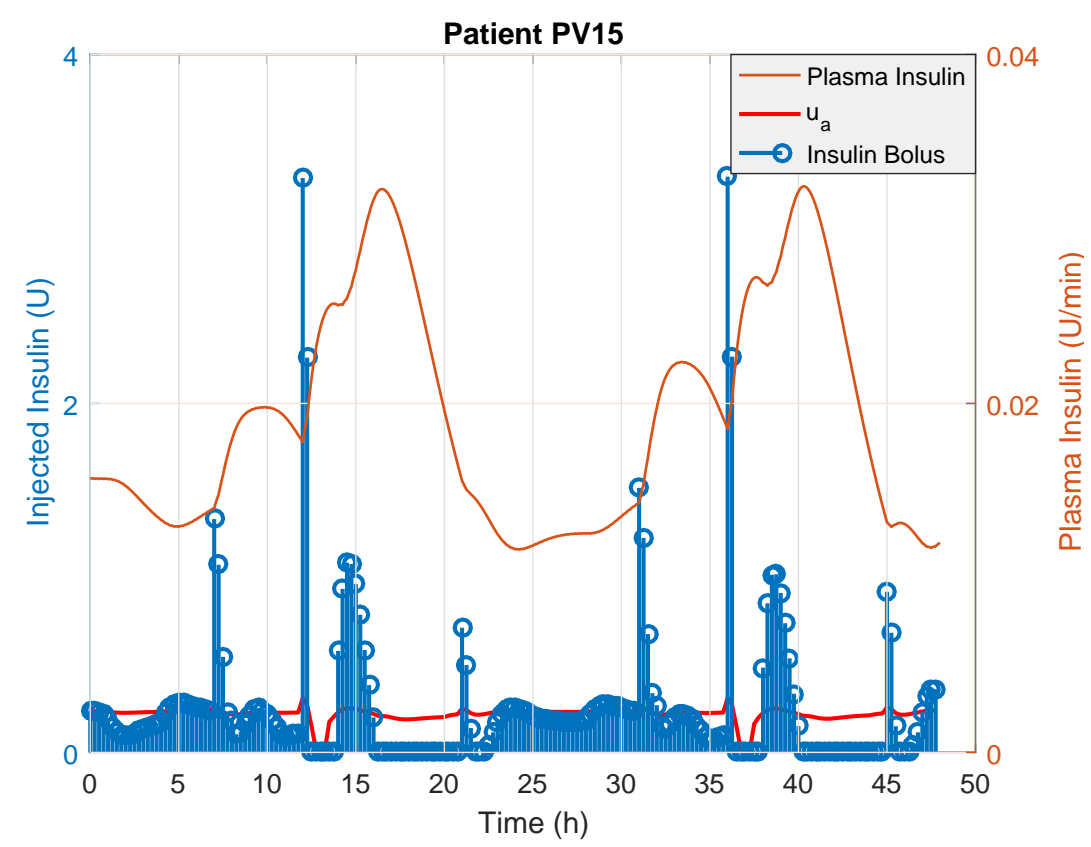

FIGURE 6 Insulin boluses (blue line) and Insulin in plasma (orange line) for patient PV15.

BG sensor is the CGM described in ${ }^{32}$, while the pump is selected as a generic one. For the first 5 hours, the basal pulse insulin value is injected to the virtual patient, to stabilize it at a given point.

Three sets of 10 patients each one - labeled as Adolescent 1 to Adolescent 10, Adult 1 to Adult 10 and Child 1 to Child 10, respectively, were simulated 5 times (a total of 50 simulations per set of patients). To increase the controllability of the system under control (i.e., to increase the controllable set to the safety blood glucose zone), a minimal basal value of $0.025(U / h)$ (or $\left.4.2 e^{-4}(\mathrm{U} / \mathrm{min})\right)$ was selected, which is the minimal value of many commercial pumps to avoid catheter obstruction. This way, given that the controller can select a range of insulin bolus values going from 0 to $25-30(\mathrm{U} / \mathrm{min})$, the total insulin injected to the virtual patient goes from $4.2 e^{-4}$ to approximately $25-30(\mathrm{U} / \mathrm{min})$, allowing almost null insulin injection to quickly avoid potential hypoglycemic episodes. Note that when a permanent non-negligible insulin basal value is used, the possibilities to compensate predicted hypoglycemic episodes are drastically reduced.

The drawback of the selected scheme is that during the fasting periods non-zero boluses are needed to keep the BG in the safe range. A benefit, on the other hand, is that no patient-dependent basal level needs to be computed, since it is the controller itself who finds the correct value that maintains the BG in a given value during the fasting periods. This characteristic is useful for time varying basal level scenarios, which is what happens in real life, within each day.

Figures 7 and 8 show the BG time evolution and the control-variability grid analysis $\left(\mathrm{CVGA}^{5}\right)$ plot corresponding to the Adolescents, while Figure 9 on the other hand, shows the insulin pulses for a particular patient (Adolescent 5). In a similar vain, Figures 10 and 11 show the BG time evolution and CVGA plot corresponding to the adults, while Figure 12 shows the insulin pulse for a particular patient (Adult 1). Finally, Figures 13 and 14 show the BG time evolution and CVGA plot corresponding to the children, while Figure 15 on the other hand, shows the insulin pulse for a particular patient (Child 1). As it can be seen, the controller was tuned with the main objective of avoiding hypoglycemic episodes, but accounting for the sufficient aggressiveness to avoid also, as long as possible, hyperglycemia. As a result, some excursions of the BG to moderate/high values can be observed after meals. However, the BG is steered back to the euglycemia zone in a relatively short time. It should be noted that the hypoglycemia is avoided without the use of any additional 'safety' methodology, such as the use of asymmetric cost functions, mechanisms of switching-off or bypassing the controller, or even the use of safety supervisory strategies. These benefits come, presumably, from the long-term accurate predictions. This can be corroborated with the results in Table 3 , where no hypoglycemia and only six hyperglycemia episodes are reported, for all patients.

${ }^{5} \mathrm{CVGA}$ are plots that easily allows one to evaluate a population test, by considering a grid in the space of upper and lower $95 \%$ confidence bounds of the BG. Red-colored region means dangerous behaviors with both hyper and hypoglycemic events. Green regions means safety behaviors. 
Table 3 shows the typical indexes used to evaluate an AP proposal. The outcome indices are reported as mean \pm standard deviation (SD) for normally distributed data and as median (interquartile range) otherwise. SD is the standard deviation, while $\mathrm{CV}$ is the coefficient of variation.

TABLE 3 Performance of the strategy

\begin{tabular}{c|l|l|l|l}
\hline & Adult patients & Child patients & $\begin{array}{l}\text { Adolescent } \\
\text { patients }\end{array}$ & All patients \\
\hline Mean BG (mg/dl) & $119.33(7.89)$ & $149.09(18.50)$ & $135.23(12.93)$ & $132.89(22.62)$ \\
SD BG (mg/dl) & $19.65 \pm 2.94$ & $38.88(11.83)$ & $29.30(21.32)$ & $27.37(19.77)$ \\
CV BG (\%) & $16.26 \pm 2.39$ & $24.15(8.18)$ & $21.55(13.28)$ & $20.94(10.76)$ \\
\hline Time percentage of BG in each zone (\%) & $0 \pm 0$ & $0 \pm 0$ \\
\hline$<54 \mathrm{mg} / \mathrm{dl}$ & $0 \pm 0$ & $0 \pm 0$ & $0 \pm 0$ & $0 \pm 0$ \\
$<60 \mathrm{mg} / \mathrm{dl}$ & $0 \pm 0$ & $0 \pm 0$ & $0 \pm 0$ & $0 \pm 0$ \\
$<70 \mathrm{mg} / \mathrm{dl}$ & $0 \pm 0$ & $52.33(26.41)$ & $67.25(16.17)$ & $67.42(24.75)$ \\
$70-140 \mathrm{mg} / \mathrm{dl}$ & $83.65(8.71)$ & $76.93 \pm 7.52$ & $87.30(19.16)$ & $89.81(21.59)$ \\
$70-180 \mathrm{mg} / \mathrm{dl}$ & $99.20(2.46)$ & $23.07 \pm 7.52$ & $12.70(19.16)$ & $10.19(21.59)$ \\
$>180 \mathrm{mg} / \mathrm{dl}$ & $0.80(2.46)$ & $3.26(3.89)$ & $0(2.33)$ & $0(3.09)$ \\
$>250 \mathrm{mg} / \mathrm{dl}$ & $0 \pm 0$ & $0(0)$ & $0 \pm 0$ & $0(0)$ \\
$>300 \mathrm{mg} / \mathrm{dl}$ & $0 \pm 0$ & $0 \pm 0$ & $0 \pm 0$ & $0 \pm 0$ \\
\hline Number of events in each zone (-) & $0 \pm 0$ & $0 \pm 0$ & $0 \pm 0$ & $0 \pm 0$ \\
\hline$<54 \mathrm{mg} / \mathrm{dl}$ & $0 \pm 0$ & $0 \pm 0$ & $0 \pm 0$ & $6(4)$ \\
$<60 \mathrm{mg} / \mathrm{dl}$ & $0 \pm 0$ & $6(2)$ & $0(2)$ \\
$<70 \mathrm{mg} / \mathrm{dl}$ & $0 \pm 0$ & $2(2)$ & $0(2)$ & $0(0)$ \\
$>180 \mathrm{mg} / \mathrm{dl}$ & $1(2)$ & $0(0)$ & $0 \pm 0$ & $32.67 \pm 21.5$ \\
$>250 \mathrm{mg} / \mathrm{dl}$ & $0 \pm 0$ & $0 \pm 0$ & $19.67 \pm 6$ & $31.16 \pm 15.83$ \\
$>300 \mathrm{mg} / \mathrm{dl}$ & $0 \pm 0.67 \pm 7$ & $33.17 \pm 16$ & $33 \pm 22$ \\
\hline
\end{tabular}

\section{6 | CONCLUSIONS}

A new Impulsive Zone MPC controller was designed based on a new impulsive affine model that accurately describes the Type I diabetic patient. In contrast with other popular models, the main features of the model considered herein that make it suitable for the proposed MPC are: i) it displays an unstable equilibrium region as the dynamics includes an integrating behavior. This equilibrium region is uniquely characterized by a set of glucose levels and one single value of insulin injection, the so-called basal rate. This permits to get a reliable description of the true T1DM patient consistent with medical practice, mainly from the point of view of the anticipative characteristics of MPC controllers. In fact, anticipating a possible unstable behavior allows the controller to take preventive actions faster. ii) It is a long-term model, and so the well-known anticipative benefits of predictive strategies is much better exploited. This way, predictable future hyper- and hypo-glycemia episodes can be avoided faster by means of a smooth insulin delivery. iii) It is an affine model, and so, no approximation is needed for the impulsive representation. Opposite to other MPC strategies, which need a permanent insulin injection (zero-order hold), here the insulin is delivered by boluses, which can prevent insulin overdoses.

The main features of the new MPC controller are: i) The use of artificial optimization variables produces a large domain of attraction. This means that disturbances that push the system away from the desired equilibrium target remain under control. ii) It works by zones in such a way that no control penalization is made when the glucose is inside the desired zone. This is not a trivial achievement, since every time the glucose is in the zone, no matter at which point it is, no unnecessary control action (insulin delivery) will be taken. iii) The selection of the main impulsive ZMPC parameters allows a variety of closed-loop 


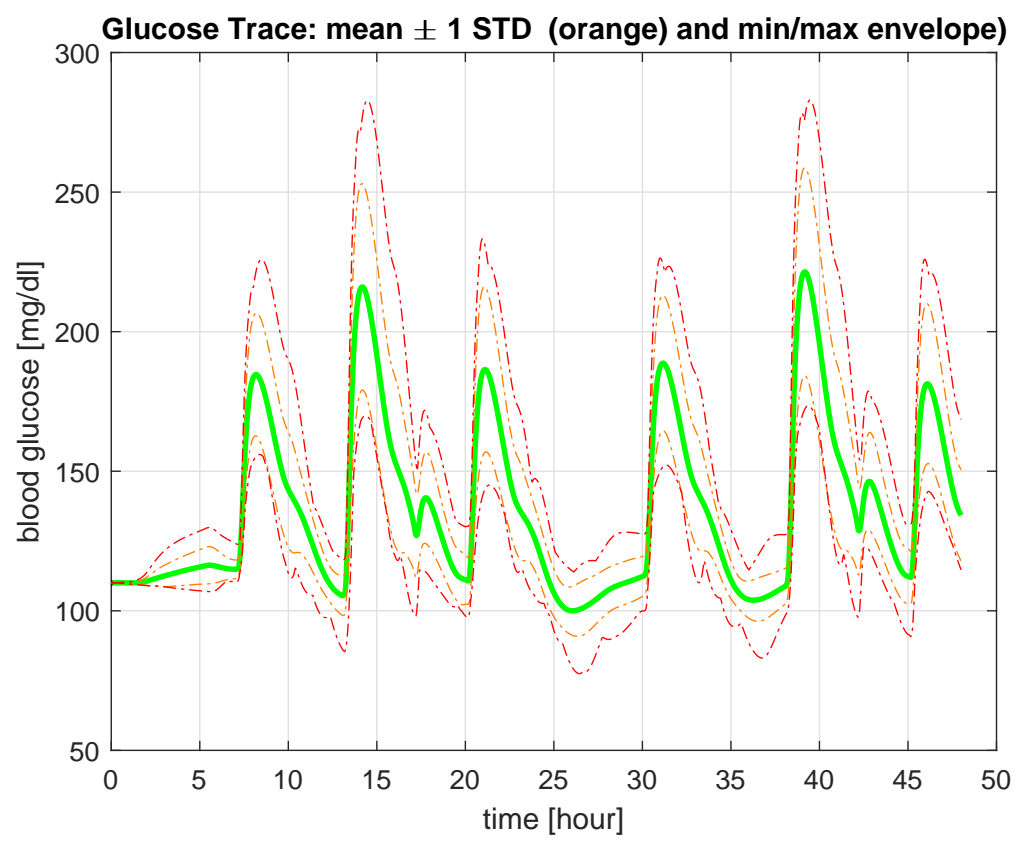

FIGURE 7 Adolescents. BG time evolution for the 50 simulations of the UVA/Padova virtual patients.

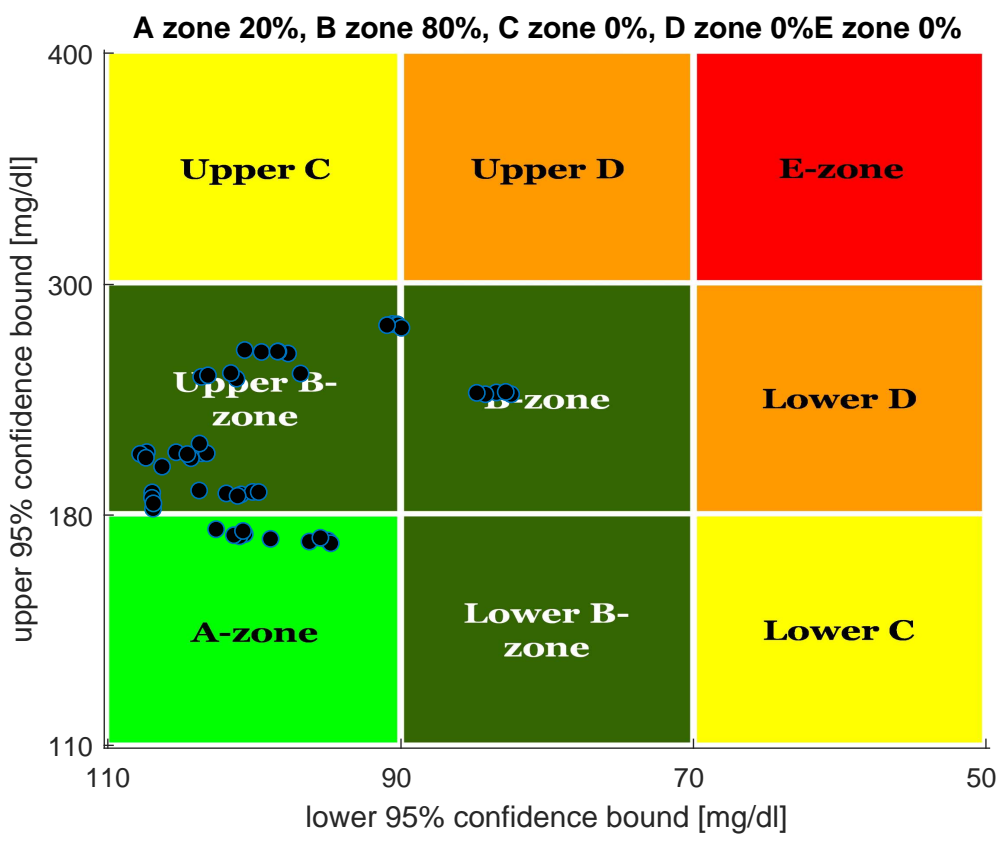

FIGURE 8 Adolescents. CVGA for the 50 simulations of the UVA/Padova virtual patients. The adolescent population presents a sparse type of subjects, with large differences in the insulin sensitivity and time constants for both, the insulin and the CHO. The controller, by a means of a personalized tuning, is able to keep any subject BG in the safety zones. No subject BG goes above $300[\mathrm{mg} / \mathrm{dll}$, while no one is below $80[\mathrm{mg} / \mathrm{dl}]$. Some of them are even in the zone between 180 and $90[\mathrm{mg} / \mathrm{dl}]$.

behaviors, and several further studies can be done to find a method to tune the controller according to the significant patient model parameters. 


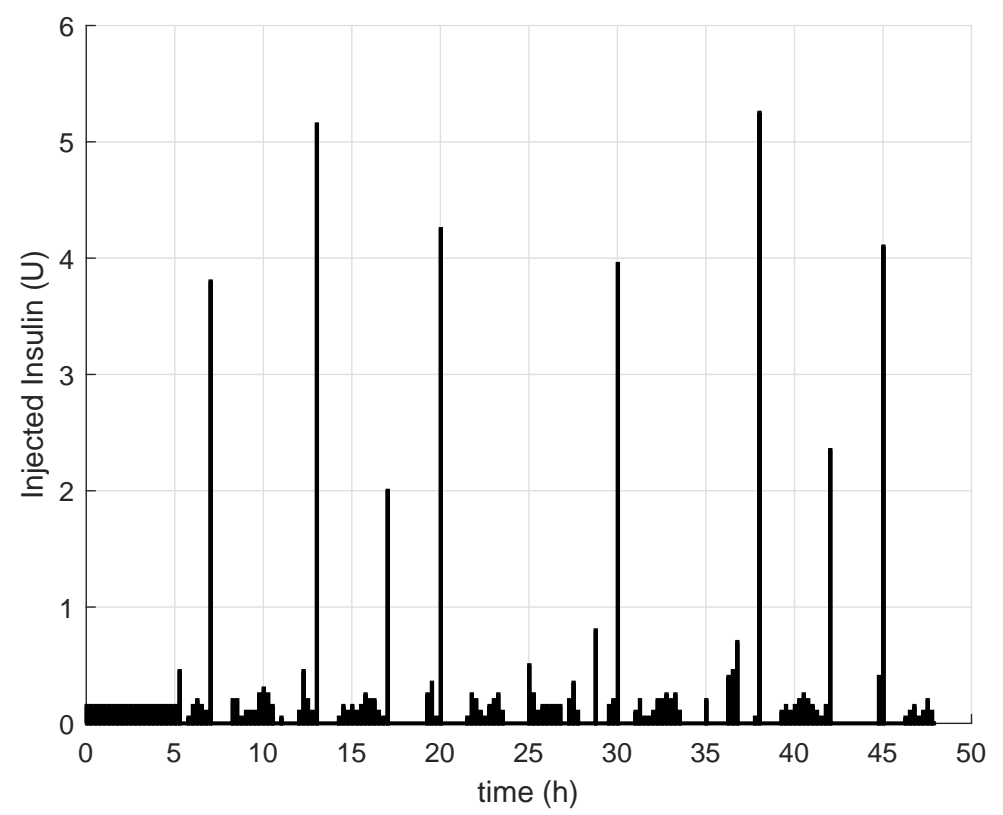

FIGURE 9 Input pulses for patient Adolescent1

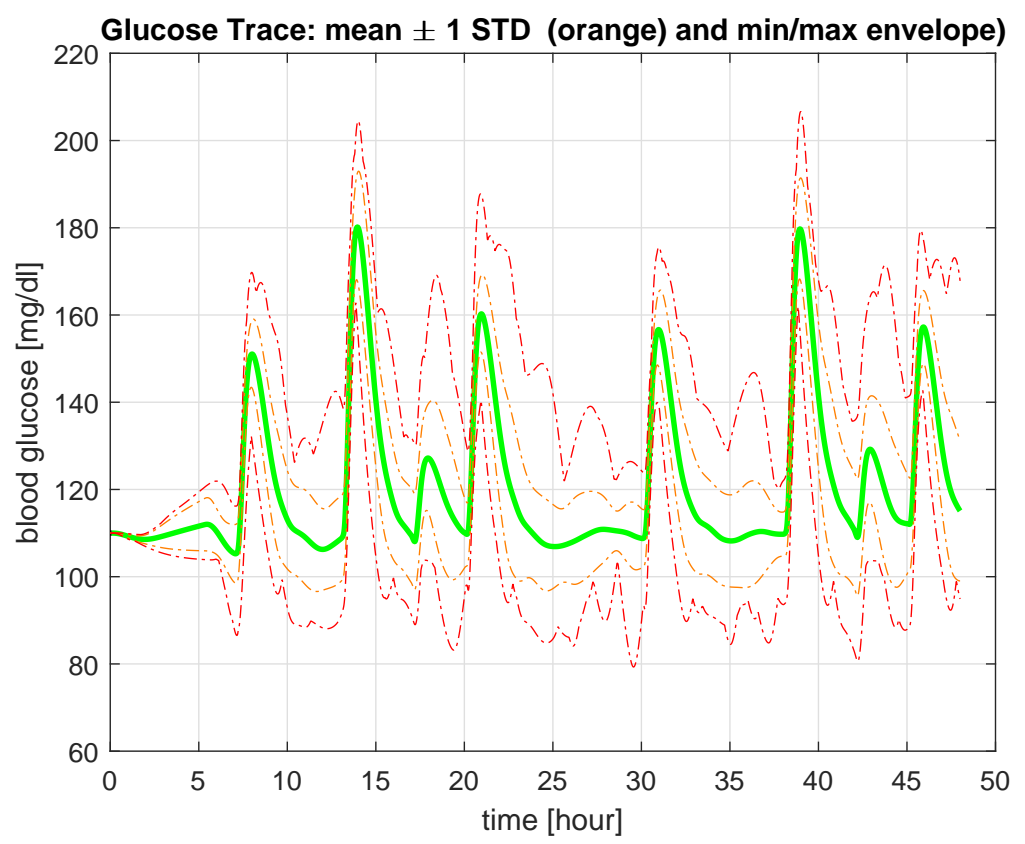

FIGURE 10 Adults. BG time evolution for the 50 simulations of the UVA/Padova virtual patients.

The testing of the proposal in more realistic scenarios is a matter of future work. The first step is to make a comparison with other available models (i.e., Bergman linearized model, 10 , Hovorka stable model, 17 , etc.) under MPC schemes. This way, conclusions can be drawn about the performance obtained with both approaches and how it is impacted by the choice of the model. 


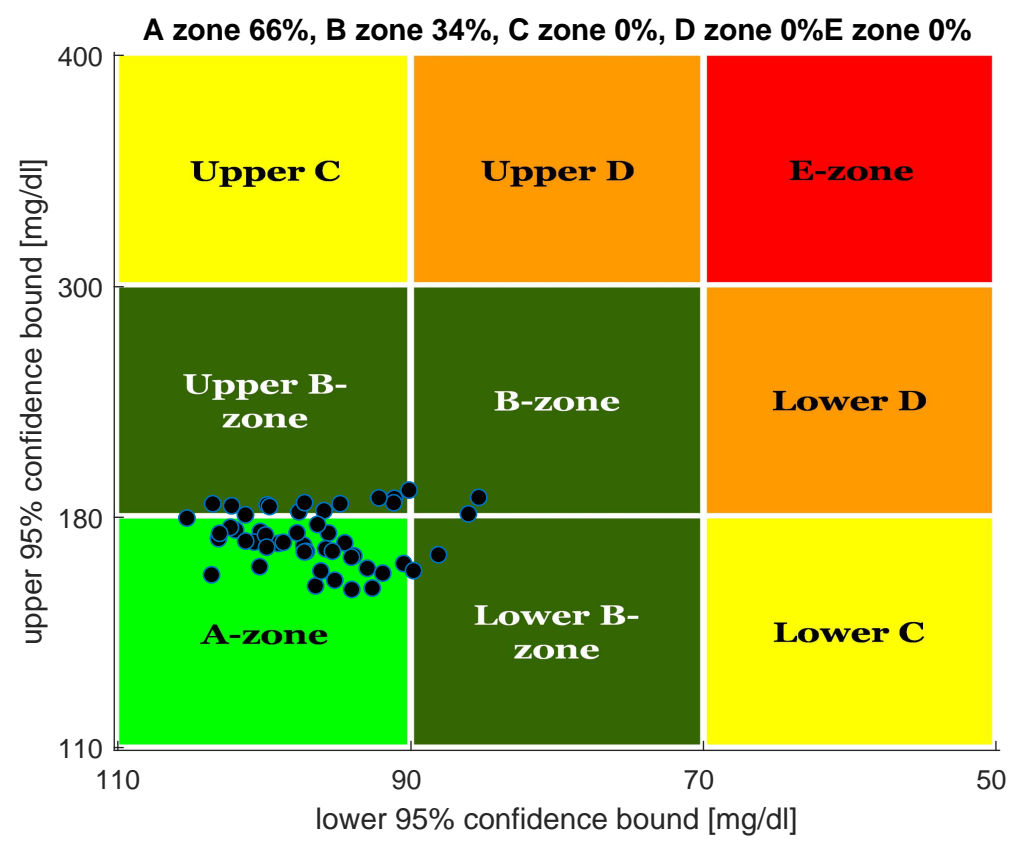

FIGURE 11 Adults. CVGA for the 50 simulations of the UVA/Padova virtual patients. The adult population is easier one to control. Accordingly, the controller is able to keep most of the subject BG in the zone between 180 and $90[\mathrm{mg} / \mathrm{dl}]$. Furthermore, no subject BG goes below $85[\mathrm{mg} / \mathrm{dll}]$.

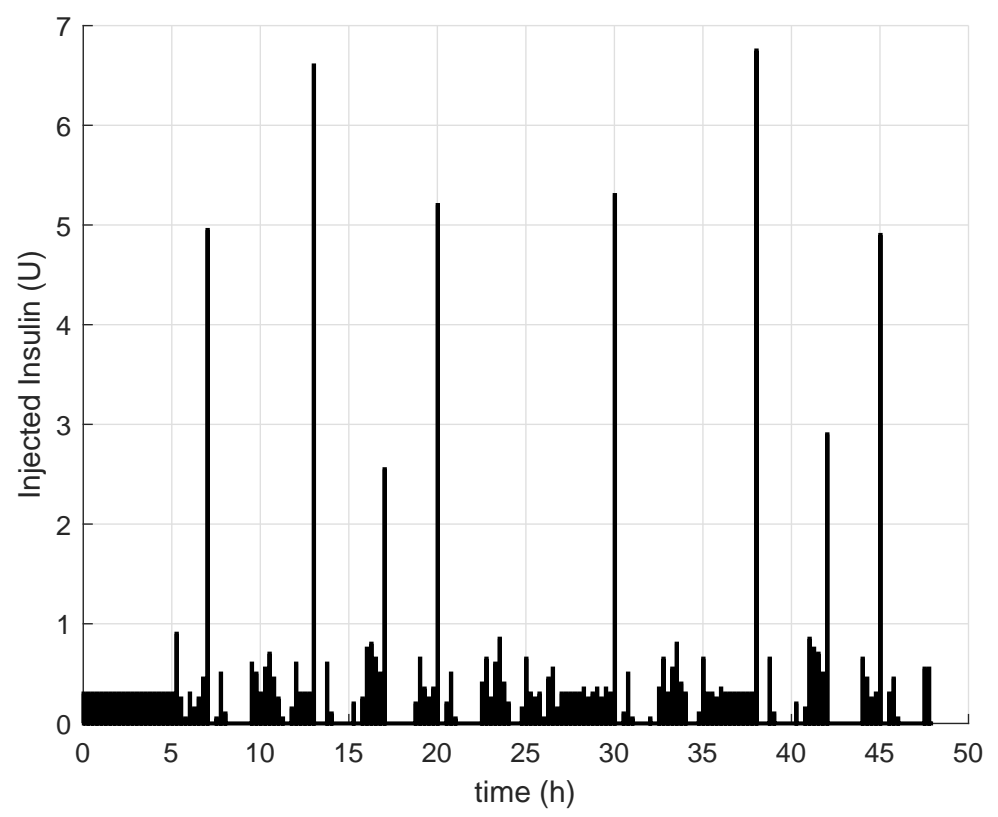

FIGURE 12 Input pulses for patient Adult1

\section{7 | ACKNOWLEDGMENT}

A. H. Gonzalez acknowledges the financial support by the Argentinean Agency of Scientific and Technological Development (ANPCyT, under the FONCyT Grant PICT-2016-3613) and the Santa Fe state Ministry of Science Technology and Productive 


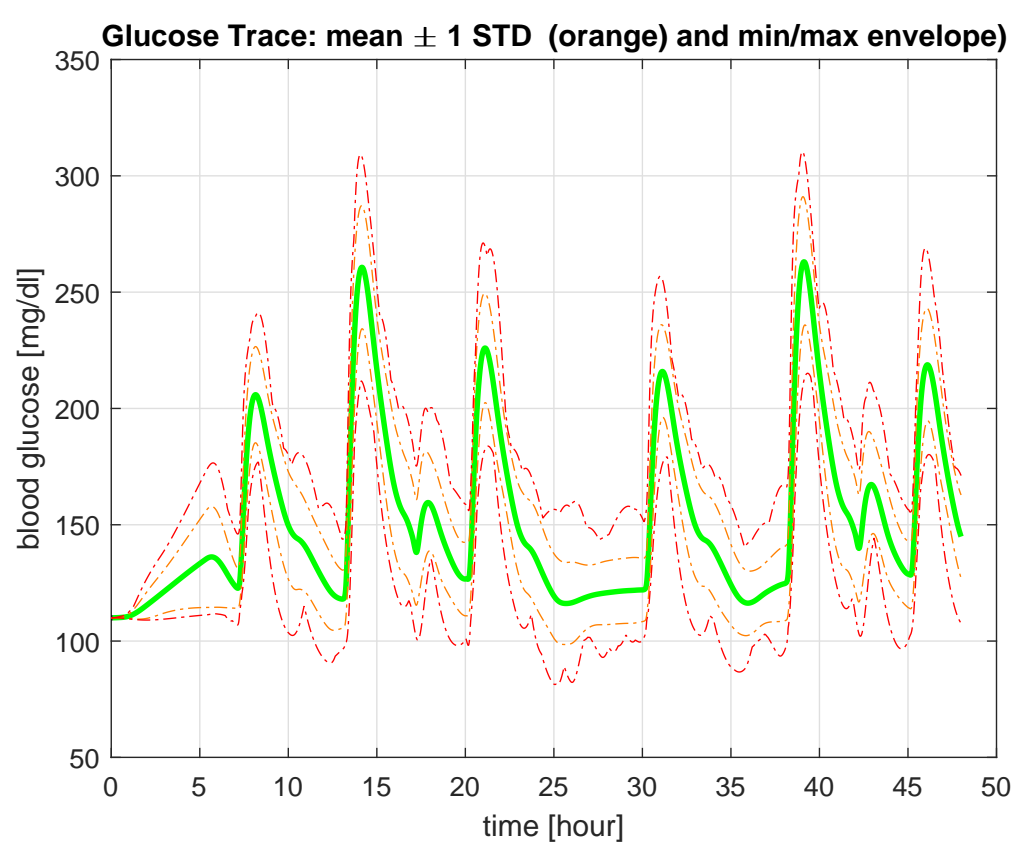

FIGURE 13 Children. BG time evolution for the 50 simulations of the UVA/Padova virtual patients.

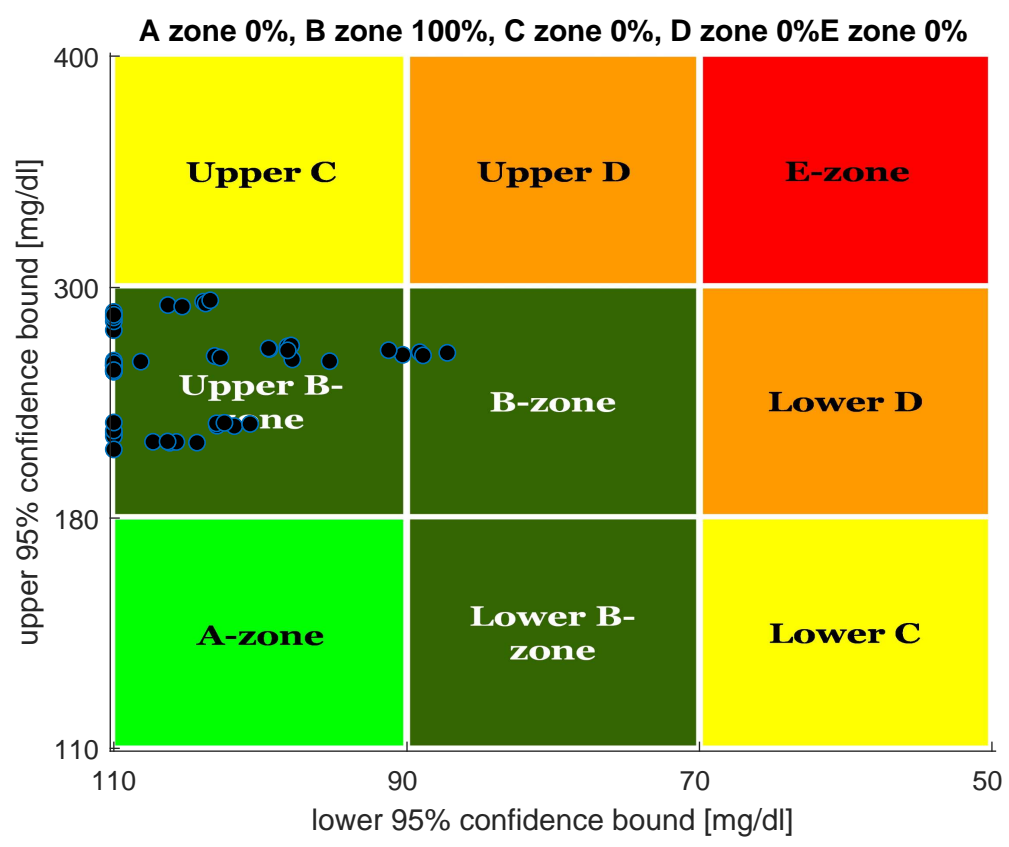

FIGURE 14 Children. CVGA for the 50 simulations of the UVA/Padova virtual patients. The children population is clearly the more challenging one. The controller is able, however, to keep all the subject BG below the upper bound of $300[\mathrm{mg} / \mathrm{dl}]$, while no one is below $83[\mathrm{mg} / \mathrm{dl}]$.

Innovation (under the ASACTeI Grant 2010-050-16). Pablo S. Rivadeneira would like to thank to the "Departamento Administrativo de Ciencia, Tecnología e Innovación (COLCIENCIAS)" from the Government of Colombia for partially supporting this work with Grant 110180763081. 


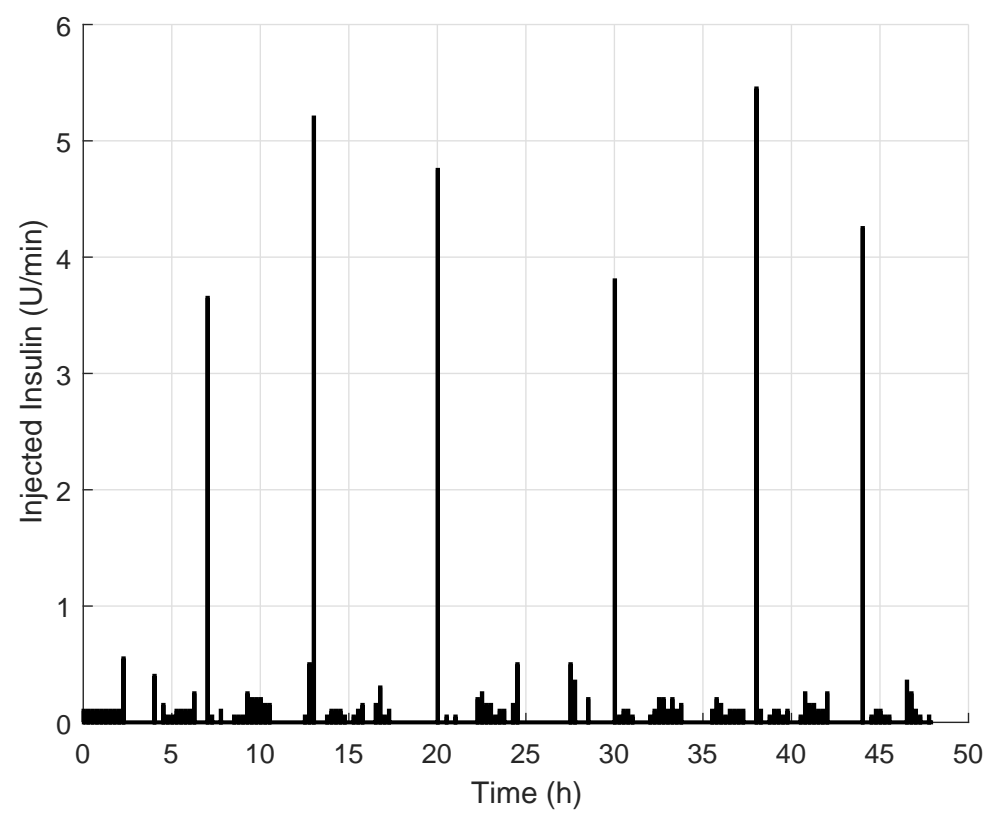

FIGURE 15 Input pulses for patient Child1

\section{8 | APPENDIX:}

\section{1 | Impulsive inputs schemes}

Consider the following affine continuous-time system

$$
\begin{aligned}
& \dot{x}(t)=A x(t)+B_{u} u(t)+B_{r} r(t)+E, \quad x(0)=x_{0}, \\
& y(t)=C x(t) .
\end{aligned}
$$

If we assume that the insulin infusion $u$ is injected to the system only at certain time instants given by $k T$, where $T$ is the fixed period, and $k \in \mathbb{N}$, then it is possible to work under the impulsive system framework recently developed in ${ }^{21}$ (see Figure 16 for a schematic plot). Formally, assume that the input is given by

$$
u(t)=u(k T) \delta(t-k T), \quad t \in[k T,(k+1) T], k \in \mathbb{N},
$$

where $\delta(t)$ is the generalized function, or distribution, Dirac impuls $e^{6}$ that fulfills $\delta(0)=\infty, \delta(t)=0$ for any $t \neq 0$, and

$$
\int_{-\infty}^{\infty} g(\zeta) \delta(\zeta) d \zeta=g(0)
$$

for any continuous, compactly supported, function $g$.

The solution of (11) at each period $k T$ can be split into two parts, the first one, describing the system in the interval $[k T, k T+$ $\Delta T$ ], and the second one describing the system in the interval $(k T+\Delta T,(k+1) T)$, for a positive and arbitrary small $\Delta T$,

${ }^{6}$ The Dirac impulse is only an abstraction to formulate the impulsive problem. The idea behind this concept is that quick insulin injections can be properly approximated by impulses, with respect to $T$. 


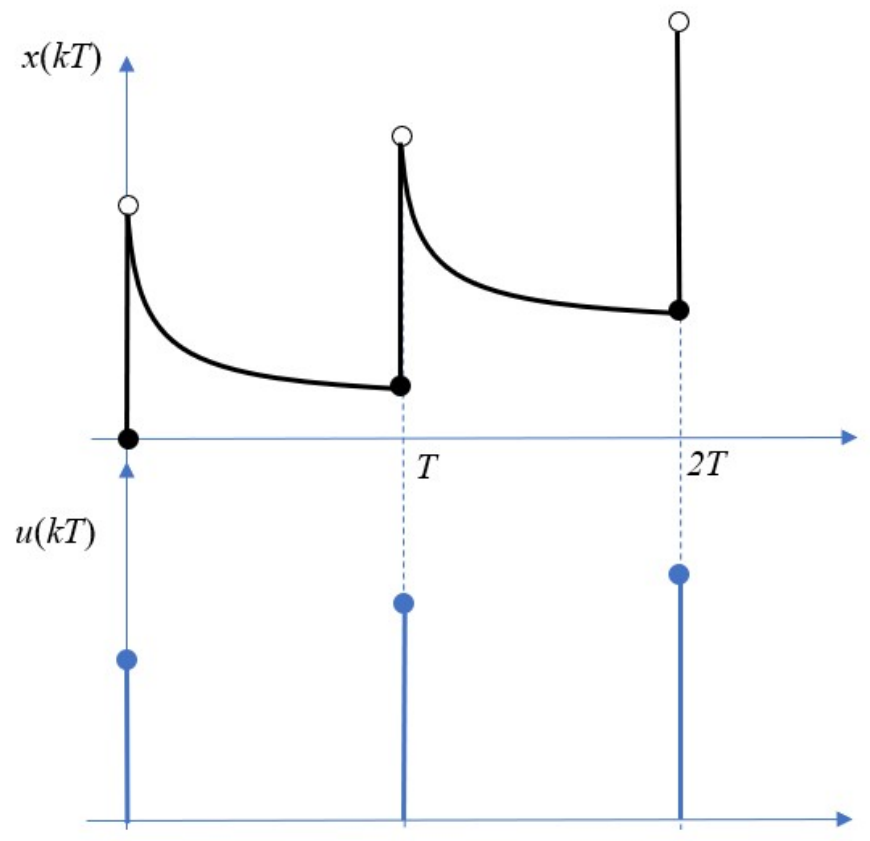

FIGURE 16 Impulsive scheme

$0<\Delta<<1$ :

$$
\begin{aligned}
\varphi(t ; x(k T), u, r) & =e^{A(t-k T)} x(k T)+\int_{k T}^{t} e^{A(t-\zeta)} B_{u} u(\zeta) \delta(\zeta-k T) d \zeta \\
& +\int_{k T}^{t} e^{A(t-\zeta)} B_{r} r(\zeta) d \zeta+\int_{k T}^{t} e^{A(t-\zeta)} d \zeta E,
\end{aligned}
$$

for $t \in[k T, k T+\Delta]$, and

$$
\begin{aligned}
\varphi(t ; x(k T+\Delta T), u, r) & =e^{A(t-k T)} x(k T+\Delta T)+\int_{k T+\Delta T}^{t} e^{A(t-\zeta)} B_{r} r(\zeta) d \zeta \\
& +\int_{k T+\Delta T}^{t} e^{A(t-\zeta)} d \zeta E,
\end{aligned}
$$

for $t \in(k T+\Delta,(k+1) T)$.

Now, if we consider the limits of this solution for $\Delta T \rightarrow 0$, it follows that

$$
x\left(k T^{+}\right) \stackrel{\Delta}{=} \lim _{\Delta T \rightarrow 0} \varphi(t ; x(k T), u, r)=x(k T)+B_{u} u(k T),
$$

where $x\left(k T^{+}\right) \stackrel{\Delta}{=} \lim _{\Delta T \rightarrow 0} x(k T+\Delta T)$, and

$$
\begin{aligned}
x(t) \triangleq \varphi\left(t ; x\left(k T^{+}\right), u, r\right) & =e^{A(t-k T)} x\left(k T^{+}\right)+\int_{k T}^{t} e^{A(t-\zeta)} B_{r} r(\zeta) d \zeta \\
& +\int_{k T}^{t} e^{A(t-\zeta)} d \zeta E,
\end{aligned}
$$


for $t \in(k T,(k+1) T)$. This latter solution is the trajectory of the continuous time system

$$
\dot{x}(t)=A x(t)+B_{r} r(t)+E, \quad x(0)=x_{0},
$$

for the aforementioned period of time.

This way, merging (12) and (13) into a single model, the following impulsive system can be obtained:

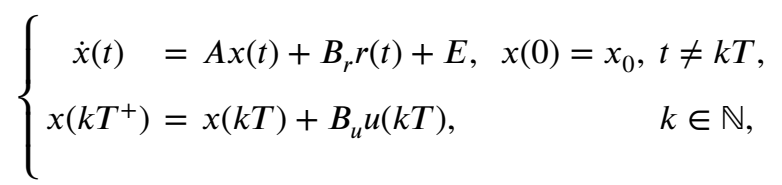

where $x\left(k T^{+}\right)$denotes the limits of $x(t)$ when $t$ approaches $k T$ from higher values. In (14), the second equation describes the discontinuities that the impulsive input causes into the system, while the first describes the free response (only affected by the disturbances $r$ ) between the discontinuities.

Remark 3. An interesting point here is that the affine impulsive system (14) has no formal equilibrium points. In fact, there is no triplet $\left(x_{s}^{i}, u_{s}^{i}, r_{s}^{i}\right)$ fulfilling the condition:

$$
\begin{aligned}
A x_{s}^{i}+B_{r} r_{s}^{i}+E & =0, \\
x_{s}^{i} & =x_{s}^{i}+B_{u} u_{s}^{i}, \quad \text { (no discontinuity), }
\end{aligned}
$$

because the first equation has no solution neither for $r_{s}^{i}=0$ (fasting condition), nor $r_{s}^{i} \neq 0$.

Consider the hypothetical case $r_{s}^{i}=r_{b}$, with $r_{b}$ a constant value. The only equilibrium in this case is $\left(x_{b} 00 r_{b} r_{b} ; 0 ; r_{b}\right)$, with $x_{b}$ any value of glycemia and $r_{b}=-\theta_{1} / \theta_{4}$, but negative values are not realistic. For the case $r_{b}=0$ (fasting condition), there is no solution. An interpretation of the latter situation is that it is not possible to maintain the system at a given point by only applying impulsive inputs, even when this input is zero. However, as it will be shown in the next section, it is possible to maintain the system (switching) inside a given region. The condition for that is: (i) to find states before and after the discontinuity that, although different, remain constant and (ii) to ensure that the transient state trajectories between these states remain inside a given set.

\section{2 | Underlying discrete-time subsystem}

A natural way to obtain a discretization (or a discrete-time system) of the continuous-time impulsive system (14) is by sampling it with a sampling time given by the period $T$ of the impulses. According to the results presented in ${ }^{29212}$, the idea is to characterize two discrete-time subsystems describing the evolution of the states $x(k T)$ and $x\left(k T^{+}\right)$; i.e., the evolution of the states before and after the discontinuities. The subsystems are as follows:

$$
\begin{gathered}
x^{\bullet}(k+1)=A^{\bullet} x^{\bullet}(k)+B_{u}^{\bullet} u^{\bullet}(k)+B_{r}^{\bullet} r^{\circ}(k)+E^{\bullet}, \quad x^{\bullet}(0)=x(0)=x_{0}, \\
x^{\circ}(k+1)=A^{\circ} x^{\circ}(k)+B_{u}^{\circ} u^{\circ}(k)+B_{r}^{\circ} r^{\circ}(k)+E^{\circ}, x^{\circ}(0)=x\left(0^{+}\right)=x_{0}+B_{u} u(0),
\end{gathered}
$$

where $x^{\bullet}(k) \stackrel{\Delta}{=} x(k T), x^{\circ}(k) \stackrel{\Delta}{=} x\left(k T^{+}\right), A^{\bullet}=A^{\circ}=e^{A T}, B_{u}^{\bullet}=e^{A T} B_{u}$ and $B_{u}^{\circ}=B_{u}$. Furthermore, the input and disturbance of each subsystem are such that $u^{\circ}(k+1)=u^{\circ}(k)=u(k T), r^{\circ}(k)=r^{\circ}(k)=r(k T)$, and $E^{\bullet}=E^{\circ} \stackrel{\Delta}{=} \int_{0}^{T} e^{A \zeta} d \zeta E, B_{r}^{\bullet}=B_{r}^{\circ} \stackrel{\Delta}{=}$ $\int_{0}^{T} e^{A \zeta} d \zeta B_{r}$.

These two subsystems are not only useful to describe the evolution of the impulsive system (14) at the sampling times $k T$ and $k T^{+}$- which is necessary to implement a MPC - but also to characterize the equilibrium regions of (14) accounting for both, the discontinuities and the free responses between them. 


\section{3 | Extended equilibrium of the impulsive system}

The idea now is to find an equilibrium set for (14), based on the underlying discrete-time subsystems (17) and (18). To do that, we first define the equilibrium pairs of (17) and (18) $-\left(x_{s}^{\circ}, u_{s}^{\circ}\right)$ and $\left(x_{s}^{\circ}, u_{s}^{\circ}\right)$, respectively - as the ones fulfilling the conditions ${ }^{7}$

$$
\begin{gathered}
x_{s}^{\circ}=A^{\bullet} x_{s}^{\circ}+B_{u}^{\bullet} u_{s}^{\bullet}+E^{\bullet}, \\
x_{s}^{\circ}=A^{\circ} x_{s}^{\circ}+B_{u}^{\circ} u_{s}^{\circ}+E^{\circ} .
\end{gathered}
$$

Given that $u^{\circ}(k+1)=u^{\circ}(k)$ by definition (and $E^{\circ}=E^{*}$ ), we have that at steady state $u_{s}^{\circ}=u_{s}^{\circ}$. This means that one equilibrium input (defined as $u_{s}^{\bullet}(k)$ for simplicity) has to fulfill both equilibrium conditions, i.e., the last equation can be written as

$$
x_{s}^{\circ}=A^{\circ} x_{s}^{\circ}+B_{u}^{\circ} u_{s}^{\circ}+E^{\circ} .
$$

This way, given that (17) and (18) are subsystems describing one single impulsive system, we need to find triplets $\left(x_{s}^{*}, x_{s}^{\circ}, u_{s}^{*}\right) \in$ $\mathcal{X} \times \mathcal{X} \times \mathcal{V}$ fulfilling both (19) and (20). Furthermore, a final condition that the equilibrium triplet $\left(x_{s}^{*}, x_{s}^{\circ}, u_{s}^{*}\right)$ has to fulfill to be a feasible extended equilibrium, is that the free responses corresponding to them (orbits) must be feasible:

$$
o_{s}\left(x_{s}^{\bullet}, u_{s}^{\bullet}\right) \in \mathcal{X}
$$

where

$$
o_{s}\left(x_{s}^{\cdot}, u_{s}^{\cdot}\right) \stackrel{\Delta}{=}\left\{\varphi\left(t ; x_{s}^{\cdot}, u_{s}^{\cdot}, 0\right), t \in(k T,(k+1) T], k \in \mathbb{N}\right\},
$$

and $\varphi\left(t ; x_{s}^{\cdot}, u_{s}^{\cdot}, 0\right)=e^{A t}\left(x_{s}+B_{u} u_{s}^{*}\right)+\int_{k T}^{t} e^{A(t-\zeta)} d \zeta E$, i.e., $\varphi(\cdot)$ it is the solution of the system [13], for an impulsive input of value $u_{s}^{*}$.

Summarizing, the extended equilibrium set $\mathcal{X}_{s}^{*}$ is characterized as 8

$$
\mathcal{X}_{s}^{\bullet} \triangleq\left\{x_{s}^{\cdot} \in \mathcal{X}: \exists u_{s}^{\cdot} \in \mathcal{V} \text { such that } A^{\bullet} x_{s}^{\cdot}+B_{u}^{\cdot} u_{s}^{\cdot}+E^{\cdot}=x_{s}^{\cdot}, o_{s}\left(x_{s}^{\cdot}, u_{s}^{\bullet}\right) \in \mathcal{X}\right\}
$$

Furthermore, it can be shown that the set of equilibrium input $9 V_{s}^{\bullet}$, is given by a singleton in the diabetes system case, i.e., $\left.V_{s}^{\cdot} \triangleq \mid \Delta u_{s}^{\cdot}\right\}=\left\{u_{b}^{\cdot}\right\}$, where $u_{b}^{\cdot}$ represents the basal insulin rate of the subsystem (17). Note that this latter impulsive equilibrium is different from the input equilibrium defined in Subsection 2.4 as the basal rate, i.e., $\left\{u_{b}^{\cdot}\right\} \neq\left\{u_{b}\right\}$. For details in the procedure to compute such equilibrium sets $\mathcal{X}_{s}^{\bullet}$ and $\mathcal{V}_{s}^{\bullet}$, in a general context, please refer to ${ }^{21}$.

\section{4 | Pulse input scheme}

Here, a pulse input scheme (i.e., when the inputs are pulses of a given duration, instead of impulses) is introduced to be consistent with the UVA/Padova simulator, in which impulsive inputs are not allowed. To properly represent model (5) under the pulse scheme, some modifications need to be made on the underlying subsystems in Subsection 8.2. Basically, matrix $B_{u}^{*}$ has to be redefined as

$$
B_{u}^{\bullet} \stackrel{\Delta}{=} e^{A(T-\Delta T)} \int_{0}^{\Delta T} e^{A(\Delta T-\zeta)} d \zeta B_{u},
$$

where $\Delta T$ represents now the duration of the input pulse. Furthermore, to characterize the extended equilibrium, matrix $B_{u}^{\circ}$ takes the form

$$
B_{u}^{\circ} \stackrel{\Delta}{=} \int_{0}^{\Delta T} e^{A(\Delta T-\zeta)} d \zeta B_{u} .
$$

This way, the extended equilibrium sets, $\mathcal{V}_{s}^{\bullet}$ and $\mathcal{X}_{s}^{\bullet}$ are different from the ones obtained in the impulsive case.

\footnotetext{
${ }^{7}$ Given that it has no sense to consider a permanent disturbance (meal), it is assumed that $r^{\bullet}=r^{\circ}=0$ in the steady state.

${ }^{8}$ Note that condition 20 is implicitly taken into account in definition 21 .

${ }^{9}$ The set of inputs for which exists an equilibrium state.
} 


\section{9 | BIBLIOGRAPHY}

\section{References}

1. Magdelaine N., Chaillous L., Guilhem I., et al. A Long-Term Model of the Glucose-Insulin Dynamics of Type 1 Diabetes. IEEE Transactions on Biomedical Engineering. 2015;62(6):1546 -1552.

2. Kadish A. H.. Automation control of blood sugar a servomechanism for glucose monitoring and control. ASAIO Journal. 1963;9(1):363-367.

3. Magni L., Raimondo D. M., Dalla Man C., De Nicolao G., Kovatchev B., Cobelli C.. Model predictive control of glucose concentration in type I diabetic patients: An in silico trial. Biomedical Signal Processing and Control. 2009;4(4):338-346.

4. Incremona G. P., Messori M., Toffanin C., Cobelli C., Magni L.. Model predictive control with integral action for artificial pancreas. Control Engineering Practice. 2018;77:86-94.

5. Messori M., Incremona G. P., Cobelli C., Magni L.. Individualized model predictive control for the artificial pancreas: In silico evaluation of closed-loop glucose control. IEEE Control Systems Magazine. 2018;38(1):86-104.

6. Grosman B., Dassau E., Zisser H. C., Jovanovic L., Doyle III F. J.. Zone model predictive control: a strategy to minimize hyper- and hypoglycemic events. Journal of Diabetes Science and Technology. 2010;4(4):961-975.

7. Gondhalekar R., Dassau E., Zisser H. C., Doyle III F. J.. Periodic-Zone Model Predictive Control for Diurnal Closed-Loop Operation of an Artificial Pancreas. Journal of Diabetes Science and Technology. 2014;7(6):1446-1460.

8. Gondhalekar R., Dassau E., Doyle III F. J.. Velocity-weighting and velocity-penalty MPC of an artificial pancreas: Improved safety and performance. Automatica. 2018;91:105-117.

9. Heusden K., Dassau E., Zisser H. C., Seborg D. E., III F. J. Doyle. Control-relevant models for glucose control using a priori patient characteristics. IEEE Transactions on Biomedical Engineering. 2011;59(7):1839-1849.

10. Bergman R. N., Phillips L. S., Cobelli C.. Physiologic evaluation of factors controlling glucose tolerance in man: measurement of insulin sensitivity and beta-cell glucose sensitivity from the response to intravenous glucose. Journal of Clinical Investigation. 1981;68(6):1456-1467.

11. Dalla Man C., Rizza R. A., Cobelli C.. Meal simulation model of the glucose-insulin system. IEEE Transactions on Biomedical Engineering. 2007;54(10):1740-1749.

12. Cobelli C., Dalla Man C., Sparacino G., Magni L., De Nicolao G., Kovatchev B. P.. Diabetes: models, signals, and control. IEEE Reviews in Biomedical Engineering. 2009;2:54-96.

13. Cobelli C., Dalla Man C., Pedersen M. G., Bertoldo A., Toffolo G.. Advancing our understanding of the glucose system via modeling: A perspective. IEEE Transactions on Biomedical Engineering. 2014;61(5):1577-1592.

14. Copp D. A., Gondhalekar R., Hespanha J. P.. Simultaneous model predictive control and moving horizon estimation for blood glucose regulation in Type 1 diabetes. Optimal Control Applications and Methods. 2017;39(2):904-918.

15. Cobelli C., Renard E., Kovatchev B.. Artificial pancreas: past, present, future. Diabetes. 2011;60(11):2672-2682.

16. Mohn A., Kavan C., Bourcelot E., Zimmermann C., Penfornis A.. Insulinothérapie fonctionnelle: Un modèle d'approche éducative pour les patients ayant un diabète de type 1. Médecine des Maladies Métaboliques. 2012;6(6):469-476.

17. Ruan Y., Wilinska M. E., Thabit H., Hovorka R.. Modeling Day-to-Day Variability of Glucose-Insulin Regulation Over 12Week Home Use of Closed-Loop Insulin Delivery. IEEE Transactions on Biomedical Engineering. 2017;64(6):1412-1419.

18. Goodwin G. C., Seron M. M., De Dona J. A.. Constrained Control and Estimation. An optimization approach. London: Springer; 2005. 
19. Limon D., Alvarado I., Alamo T., Camacho E. F.. MPC for tracking of Piece-Wise Constant References for Constrained Linear Systems. Automatica. 2008;44(9):2382-2387.

20. Ferramosca A., Limon D., González A. H., Odloak D., Camacho E. F.. MPC for tracking zone regions. Journal of Process Control. 2010;20(4):506-516.

21. Rivadeneira P. S., Ferramosca A., González A. H.. Control Strategies for Non-zero Set-point Regulation of Linear Impulsive Systems. IEEE Transactions on Automatic Control. 2018;69(9):2994-3001.

22. Tolic H. M., Mosekilde E., Stuirs Jepe. Modeling the insulin-glucose feedback system: the significance of pulsatile insulin secretion. Journal of Theoretical Biology. 2000;207:361-375.

23. Garcia-Tirado J., Corbett J. P., Boiroux D., Jørgensen J. B., Breton M. D.. Closed-loop control with unannounced exercise for adults with type 1 diabetes using the Ensemble Model Predictive Control. Journal of Process Control. 2019;80:202-210.

24. Ljung L.. System identification: theory for the user. Upper Saddle River, NJ: Prentice Hall; 2th edition ed.1999.

25. Messori M., Toffanin C., Del Favero S., Nicolao G. De, Cobelli C., Magni L.. Model individualization for artificial pancreas. Computer methods and programs in biomedicine. 2019;171:133-140.

26. Lee J. B., Dassau E., Gondhalekar R., Seborg D. E., Pinsker J. E., III F. J. Doyle. Enhanced model predictive control (eMPC) strategy for automated glucose control. Industrial \& engineering chemistry research. 2016;55(46):11857-11868.

27. Boiroux D., Duun-Henriksen A. K., Schmidt S., et al. Overnight glucose control in people with type 1 diabetes. Biomedical Signal Processing and Control. 2018;39:503-512.

28. Gondhalekar R., Dassau E., Doyle III F. J.. Tackling problem nonlinearities and delays via asymmetric, state-dependent objective costs in MPC of an artificial pancreas. IFAC-PapersOnLine. 2015;23(48):154-159.

29. Rivadeneira P. S., Ferramosca A., González A. H.. MPC with state window target control in linear impulsive systems. IFAC-PapersOnline. 2015;48(23):507-512.

30. Ferramosca A., Limon D., González A. H., Alvarado I., Camacho E. F.. Robust MPC for tracking zone regions based on nominal predictions. Journal of Process Control. 2012;22(10):1966-1974.

31. Godoy J. L., Abuin P., Rivadeneira P. S., González A. H.. Modeling, identification and state estimation for artificial pancreas. Part I. In:Proceedings of the 26th Congreso Argentino de Control Automático (AADECA). 2018;. Buenos Aires, Argentina.

32. Vettoretti M., Facchinetti A., Sparacino G., Cobelli C.. Type-1 Diabetes Patient Decision Simulator for In Silico Testing Safety and Effectiveness of Insulin Treatments. IEEE Transactions on Biomedical Engineering. 2017;65(6):1281-1290.

33. Doyle III F. J.. Zone Model Predictive Control of an Artificial Pancreas. In: Proceedings of the 10th World Congress on Intelligent Control and Automation. 2012;. Beijing, China.

34. Kovatchev D. T., Breton M., Dalla Man C., Cobelli C.. A proof of concept in closed-loop control of type 1 diabetes. Journal of Diabetes Science and Technology. 2009;3:44-45. 